\title{
"Alpen-Demokratie«? \\ Die Herausforderung demokratischer Legitimität in der makroregionalen Strategie für den Alpenraum
}

\author{
Melanie Plangger
}

\section{Einleitung}

Grenzüberschreitende Kooperation ${ }^{1}$ hat sich in den letzten Jahrzehnten von einem Nischenthema zu einem eigenständigen Politikbereich der EU entwickelt. Mit der Schaffung finanzieller Unterstützungsprogramme und rechtlicher Möglichkeiten hat sich die Anzahl grenzüberschreitender Verbünde in der EU stark erhöht. ${ }^{2}$ Zugleich hat Kooperation an Qualität gewonnen. Grenzüberschreitende Strukturen stellen heute nicht nur einen Rahmen für die Diskussion gemeinsamer Probleme dar, sondern entwerfen über Projekte sowie politische Deklarationen mittel- und langfristige Entwicklungsvisionen. Die dabei entstehenden Netzwerke mobilisieren EU-Fördermittel, ${ }^{3}$ beeinflussen Politiken ${ }^{4}$ und verhandeln Beziehungen zwischen den territorialen Ebenen. ${ }^{5}$ Damit wird die Teilnahme in grenz-

1 Unter grenzüberschreitender Kooperation wird in diesem Beitrag die Zusammenarbeit von subnationalen öffentlichen Gebietskörperschaften, also Regionen, Städten und Gemeinden, über nationalstaatliche Grenzen hinweg verstanden. Die EU fasst mit dem Begriff grenzüberschreitende Kooperation die kleinräumige Kooperation von benachbarten Regionen und Städten, während größere geographische Verbünde unter den Begriff transnationale Kooperation fallen. Territoriale Kooperation dient in der EU als Überbegriff für klein- und großräumige grenzüberschreitende Strukturen.

2 Markus Perkmann, „Cross-Border Regions in Europe: Significance and Drivers of Regional Cross-Border Co-Operation«, (2003) 10 European Urban and Regional Studies 153.

3 Ebd., 167.

4 Franziska Sielker und Jörg Mirtl, »Positioning EU Macro-Regions: When Sectoral Policies Meet Cohesion Policy«, (2017) 5 European Structural \& Investment Funds Journal 223.

5 Joachim Blatter, »Grenzüberschreitende Regionenbildung und Europäische Integration. Erkenntnisse aus einem transatlantischen Vergleich«, in Thomas Conzelmann und Michèle Knodt (Hrsg.), Regionales Europa - Europäisierte Regionen (Campus, 2002). 
überschreitenden Kooperationen auch zunehmend zum Kriterium für Einfluss und finanzielle Möglichkeiten. ${ }^{6}$

Die Entwicklung grenzüberschreitender Kooperation weg vom Experimentier- hin zum relevanten Politikfeld wirft Fragen nach der Legitimität grenzüberschreitenden Handelns auf. Stand lange Zeit vor allem die Funktionalität grenzüberschreitender Zusammenarbeit im Vordergrund, nimmt die wissenschaftliche Beschäftigung mit Fragen der Demokratie und Legitimität zu. ${ }^{7}$ Im Mittelpunkt steht dabei die Frage, inwiefern grenzüberschreitende Entscheidungen und Handlungen demokratischen Normen entsprechen und damit als legitim, oder "normativ richtig «, gelten können. Viele Untersuchungen zeigen, dass grenzüberschreitende Kooperation die Voraussetzungen für demokratisches Handeln nicht erfüllt. ${ }^{8}$ Zugleich werfen sie Fragen nach den möglichen Grenzen bestehender Demokratiemodelle im Kontext grenzüberschreitender Kooperation auf.

Der vorliegende Beitrag beschäftigt sich mit der demokratischen Legitimität grenzüberschreitender Kooperation. Ziel des Beitrags ist es, die Problemstellung vor dem Hintergrund unterschiedlicher Vorstellungen von demokratischer Legitimität zu beleuchten. Als empirisches Beispiel dient dabei die makroregionale Strategie für den Alpenraum, die als jüngstes EU-Kooperationsinstrument sieben Staaten und 48 Regionen in einen grenzüberschreitenden Rahmen einbettet. Das erste Kapitel beschäftigt sich mit dem Konzept demokratischer Legitimität. Dabei stellt der Beitrag mehrere Demokratiemodelle und ihre Vorstellungen von Legitimität gegenüber. Der Beitrag unterscheidet repräsentative Demokratiemodelle, die die Repräsentation von territorial oder funktional definierten Perso-

6 Franziska Sielker, »A Stakeholder-Based EU Territorial Cooperation: The Example of European Macro-Regions«, (2016) 24 European Planning Studies 1995.

7 Olivier Kramsch und Virginie Mamadouh, "Crossing Borders of Political Governance and Democracy«, (2003) 18 Journal of Borderlands Studies 39; vgl. Patrik Hall, »Opportunities for Democracy in Cross-Border Regions? Lessons from the Øresund Region«, (2008) 42 Regional Studies 423; Markus Kratochwill und Martin Fröhlich, »Legitimität und demokratische Potenziale grenzüberschreitender Governance. Die Institutionen der Euregio Bodensee«, in Philippe Koch und Urs Scheuss (Hrsg.), Governance und Demokratie: Vier Fallstudien eines Forschungsseminars (National Centre of Competence in Research, University of Zurich, 2008).

8 Hall, »Opportunities for Democracy« (Fn. 7); Kratochwill und Fröhlich, »Legitimität und demokratische Potenziale« (Fn. 7); André Remy Berberich und Jörg Röber, »Demokratiedefizite europäischer Grenzregionen: Legitimation und Partizipation in transnationalen Räumen«, in Stephan A. Jansen, Eckhard Schröter und Nico Stehr (Hrsg.), Bürger. Macht. Staat? Neue Formen gesellschaftlicher Teilhabe, Teilnahme und Arbeitsteilung (Springer VS, 2012). 
nenkreisen in den Mittelpunkt stellen, von Demokratiemodellen, die eine direkte, möglichst breite oder deliberative Beteiligung am Entscheidungsprozess betonen.

Das zweite und das dritte Kapitel ziehen die dargestellten Demokratiemodelle zur empirischen Untersuchung der Alpenraumstrategie heran. Die Alpenraumstrategie eignet sich als neuer Kooperationsrahmen, der Einfluss auf das Macht- und Politikgefüge in der EU nimmt, besonders gut dazu, Fragestellungen der Legitimität zu behandeln. Der Beitrag beleuchtet dabei Potenziale und Grenzen des Anspruchs, demokratische Normen auf die grenzüberschreitende Sphäre zu übertragen. Ein abschließendes Fazit fasst die vorgebrachten Argumente zusammen und stellt Schlussfolgerungen zum Verhältnis zwischen grenzüberschreitender Kooperation und demokratischer Legitimität an.

\section{Das Konzept demokratischer Legitimität}

Politische Entscheidungen werden in der Regel von der Bevölkerung als verbindlich akzeptiert. Dies gilt auch dann, wenn politische Entscheidungen den Interessen der Bürger zuwiderlaufen oder ihnen Pflichten aufbürden. Dabei stützt sich diese Akzeptanz nicht nur auf den Sanktions- und Polizeiapparat einer politischen Einheit. Vielmehr erkennen alle Beteiligten die Autorität, die ihnen gegenüber angewandt wird, als gültig oder gerechtfertigt an, ${ }^{9}$ da die Art und Weise, in der Autorität erworben und ausgeführt wird, bestimmten, allgemein anerkannten Normen entspricht. ${ }^{10}$ Sie erscheint den Beteiligten daher normativ richtig. ${ }^{11}$ Diese generelle Akzeptanz wird mit dem Begriff Legitimität gefasst. Legitimität ist damit die Prozedur, die eine Entscheidung annehmbar und angemessen macht. ${ }^{12}$ Sie wird meist mit Demokratie verbunden und impliziert damit die Über-

9 Victor Bekkers und Arthur Edwards, "Legitimacy and Democracy: A Conceptual Framework for Assessing Governance Practices", in Victor Bekkers et al. (Hrsg.), Governance and the Democratic Deficit: Assessing the Democratic Legitimacy of Governance Practices (Ashgate, 2007), 48.

10 David Beetham und Christopher Lord, Legitimacy and the EU (Routledge, 2013), 3.

11 Philippe C. Schmitter, "Governance in the European Union. A Viable Mechanism for Future Legitimation?", in Arthur Benz und Yannis Papadopoulos (Hrsg.), Governance and Democracy: Comparing National, European and International Experiences (Routledge, 2006), 159.

12 Bernard Manin, Elly Stein und Jane Mansbridge, »On Legitimacy and Political Deliberation«, (1987) 15 Political Theory 338, 363. 
einstimmung von Autorität mit demokratischen Normen und Werten. ${ }^{13}$ Dies bedeutet insbesondere, dass Entscheidungen von den authentischen Präferenzen der Bevölkerung abgeleitet sind und von dieser kontrolliert werden können. ${ }^{14}$

Das Konzept demokratischer Legitimität betont damit, dass es nicht ausreicht, Politiken zu gestalten, die positive Auswirkungen für die Bürger haben. Diejenigen, die Politik machen, sowie der grundsätzliche Prozess der Politikgestaltung und -entscheidung, müssen ebenso wie die Resultate von den Bürgern akzeptiert und als normativ richtig, also bestimmten, demokratischen Normen folgend, anerkannt werden. Ansonsten laufen auch Politiken, die sehr günstige Auswirkungen haben, Gefahr, nicht akzeptiert zu werden. In der Literatur werden daher häufig drei Dimensionen von Legitimität - Input-Legitimität, Throughput-Legitimität und OutputLegitimität - unterschieden. ${ }^{15}$ Eine erste Dimension, Input-Legitimität, bezieht sich auf die konkreten Möglichkeiten Interessen und Wünsche in den Politikprozess einzubringen. Die zweite Dimension, ThroughputLegitimität, beschäftigt sich mit dem Prozess der Entscheidungsfindung, den Akteuren, die an Entscheidungen mitwirken, ihren Möglichkeiten, auf Entscheidungen Einfluss zu nehmen, und möglichen Asymmetrien im Entscheidungsprozess. Die dritte Dimension, Output-Legitimität, behandelt die konkreten Auswirkungen der Entscheidungen. Sie beschäftigt sich damit, inwiefern ursprünglich formulierte Ziele realisiert wurden, welche Effekte die gesetzten Maßnahmen zeitigen, wie die Resultate mit den Interessen und Wünschen der Bürger korrespondieren und wie Bürger Ergebnisse kontrollieren und sanktionieren können. Tabelle 1 illustriert die Mechanismen der drei Dimensionen demokratischer Legitimität.

13 Vgl. Bekkers und Edwards, "Legitimacy and Democracy« (Fn. 9); Anders Uhlin, »Democratic Legitimacy of Transnational Actors: Mapping Out the Conceptual Terrain«, in Eva Erman und Anders Uhlin (Hrsg.), Legitimacy Beyond the State? Re-Eexamining the Democratic Credentials of Transnational Actors (Palgrave Macmillan, 2010).

14 Arthur Benz, »Postparlamentarische Demokratie? Demokratische Legitimation im kooperativen Staat«, in Michael T. Greven (Hrsg.), Demokratie - eine Kultur des Westens? 20. Wissenschaftlicher Kongress der Deutschen Vereinigung für Politische Wissenschaft (Leske + Budrich, 1998), 202.

15 Vgl. Fritz W. Scharpf, »Demokratische Politik in der internationalisierten Ökonomie«, in Greven (Hrsg.), Demokratie - eine Kultur des Westens? (Fn. 14); Bekkers und Edwards, »Legitimacy and Democracy« (Fn. 9), 57 ff; Joachim Blatter, »Demokratie und Legitimation«, in Arthur Benz et al. (Hrsg.), Handbuch Governance. Theoretische Grundlagen und empirische Anwendungsfelder (VS Verlag für Sozialwissenschaften, 2007). 
Tabelle 1: Die drei Dimensionen demokratischer Legitimität

\begin{tabular}{ll}
\hline Input-Legitimität & Möglichkeiten, Interessen einzubringen \\
Throughput-Legitimität & Mitwirkung an und Gestaltung des Entscheidungsprozesses \\
Output-Legitimität & $\begin{array}{l}\text { Resultate der Zusammenarbeit, Kontroll- und Sanktionsme- } \\
\text { chanismen }\end{array}$ \\
\hline
\end{tabular}

Dabei gibt es allerdings kein allgemein anerkanntes Schema, welche Bedingungen Systeme erfüllen müssen, um demokratisch legitim zu sein. Vielmehr betonen unterschiedliche Demokratiemodelle auch unterschiedliche Voraussetzungen. ${ }^{16}$ Modelle, die Repräsentation in den Vordergrund stellen, untersuchen vor allem, wie Vertreter verantwortlich gehalten werden können und Interessen aggregieren und einbringen. Dabei kann das Modell territorialer Repräsentation, das auf der Wahl von Vertretern territorialer Wahlkreise beruht, vom Modell funktionaler Repräsentation, das die Bedeutung von Interessengruppen hervorhebt, unterschieden werden. Modelle, die stärker auf Teilhabe abzielen, prüfen die Möglichkeiten breiter Bevölkerungskreise, effektiv an Entscheidungen und Prozessen mitzuwirken. Direkte Demokratiemodelle zielen auf die direkte Beteiligung an Entscheidungen, etwa in Form von Abstimmungen, ab. Partizipative Demokratiemodelle betonen die möglichst breite Einbindung aller Betroffenen, während deliberative Modelle den rationalen, verständnisbasierten Diskussionsprozess in den Mittelpunkt stellen.

Legitimität ist kein rein akademisches Konzept, sondern hat zur Folge, dass alle Beteiligten bestimmte Entscheidungen und Prozesse anerkennen, akzeptieren und einhalten. Auch grenzüberschreitende Kooperation steht vor der Herausforderung, Legitimität für Entscheidungen und Handlungen zu schaffen. Zwar entstehen durch grenzüberschreitende Kooperation zumeist keine neuen Regeln und Gesetze, Kooperation wirkt aber auf bestehende Regeln und Institutionen ein. Es werden thematische Prioriäten für die grenzüberschreitende Entwicklung festgelegt, Regeln über Grenzen hinweg koordiniert und konkrete Projekte, die der Implementierung der gemeinsam definierten Prioritäten dienen, realisiert.

Ein Beispiel für diesen Einfluss sind makroregionale Strategien. ${ }^{17} \mathrm{Ma}-$ kroregionale Strategien sind nach den finanziellen Unterstützungspro-

16 Vgl. Manfred G. Schmidt, Demokratietheorien. Eine Einfübrung (5. Aufl., Springer VS, 2010); Peter Massing, Gotthard Breit und Hubertus Buchstein (Hrsg.), Demokratietheorien. Von der Antike bis zur Gegenwart (9. Aufl., Wochenschau Verlag, 2017).

17 Vgl. Jonathan Metzger und Peter Schmitt, »When Soft Spaces Harden: The EU Strategy for the Baltic Sea Region«, (2012) 44 Environment and Planning A 263; 
grammen, von denen INTERREG das Prominenteste ist, und dem rechtlichen Instrument Europäischer Verbund für Territoriale Zusammenarbeit (EVTZ) der dritte EU-Rahmen für grenzüberschreitende Kooperation. Bislang wurden vier makroregionale Strategien im Ostseeraum, im Donauraum, in der Region Adriatisch-Ionisches Meer und im Alpenraum geschaffen. Um eine stärker politisch-strategische Dimension zu gewährleisten, beruhen makroregionale Strategien auf einem Strategiedokument, das klare Prioritäten für die Zusammenarbeit definiert. Das Strategiedokument sowie ein Aktionsplan werden von der Europäischen Kommission erarbeitet und vom Europäischen Rat bestätigt. Konkret implementiert werden die Prioritätsbereiche dann im Rahmen von Steuerungs- oder Aktionsgruppen, in denen vor allem Mitglieder der öffentlichen Verwaltungen Projekte ausarbeiten und Erfahrungen, Probleme und Lösungen austauschen. Dabei ist auch vorgesehen, dass sich die Politiker auf makroregionaler Ebene regelmäßig abstimmen, um die politische Umsetzung zu erleichtern.

Obwohl makroregionale Strategien keine neuen Fördermittel, Institutionen oder Regeln schaffen sollen, haben sie damit einen potentiell weitreichenden Einfluss. Makroregionale Strategien vereinen vielfältige Akteure in neuen, grenzüberschreitenden Netzwerken und definieren thematische Prioritäten für die grenzüberschreitende Entwicklung. Sie nehmen damit auch Einfluss auf EU-, nationale und regionale Politiken. ${ }^{18}$ Ihre direkte Verankerung in den europäischen Institutionen und die begleitende Teilnahme der Europäischen Kommission erleichtern den Zugang zu EUFördermitteln. ${ }^{19}$ Daneben bieten sie ein Forum für die Neuverhandlung etablierter Beziehungen zwischen den territorialen Ebenen. ${ }^{20}$ Makroregionale Strategien haben damit redistributive und regionalpolitische Auswirkungen, die Fragen nach der allgemeinen Akzeptanz makroregionalen Handelns aufwerfen.

Sielker, »A Stakeholder-Based EU Territorial Cooperation«(Fn. 6); Stefan Gänzle, »Macro-Regional Strategies of the European Union (EU) and Experimentalist Design of Multi-Level Governance: The Case of the EU Strategy for the Danube Region«, (2017) 27 Regional \& Federal Studies 1.

18 Sielker, "A Stakeholder-Based EU Territorial Cooperation« (Fn. 6).

19 Dominic Stead, Franziska Sielker und Tobias Chilla, »Macro-Regional Strategies: Agents of Europeanization and Rescaling? «, in Stefan Gänzle und Kristine Kern (Hrsg.), A >Macro-Regional Europe in the Making: Theoretical Approaches and Empirical Evidence (Palgrave Mcmillan, 2016), 112.

20 Melanie Plangger, "Building Something Beautiful with Stones: How Regions Adapt to, Shape and Transform the EU Opportunity Structure«, (2017) Regional \& Federal Studies 1. 
Der vorliegende Beitrag beleuchtet die demokratische Legitimität grenzüberschreitender Kooperation am Beispiel der vierten makroregionalen Strategie, die für den Alpenraum geschaffen wurde. Die Alpenraumstrategie wurde im Jänner 2016 in die Implementierung überführt, ihr Entstehungsprozess reicht allerdings einige Jahre zurück. Der Anstoß für die Alpenraumstrategie kam vor allem von den Alpenregionen, ${ }^{21}$ also den österreichischen und deutschen Bundesländern, den italienischen Regionen und Provinzen, den französischen Regionen sowie den Schweizer Kantonen. Regionale Politiker forderten bereits im Jahr 2010, und damit ein Jahr nach Schaffung der ersten Strategie im Ostseeraum, ${ }^{22}$ eine eigene makroregionale Strategie für den Alpenraum. ${ }^{23}$ Im Anschluss erarbeiteten die Regionen ein erstes inhaltliches Papier, das im Jahr 2012 von 26 Regionen des Alpenraums auf politischer Ebene verabschiedet wurde. ${ }^{24}$ In der Folge gewannen die Regionen die Unterstützung ihrer nationalen Regierungen, die gemeinsam mit den Regionen im Oktober 2013 für eine makroregionale Strategie eintraten. ${ }^{25}$ Die nationalen Regierungen trugen das Thema daraufhin in den Europäischen Rat, der die Europäische Kom-

21 Der Begriff »Region « kann verschiedene administrative, symbolische oder territoriale Konfigurationen repräsentieren. In diesem Beitrag wird der Begriff primär nach dem NUTS (Nomenclature des unités territoriales statistiques) 2-Schema der $\mathrm{EU}$, das italienische und französische Regionen oder deutsche Bundesländer als Grundeinheiten makroregionaler Strategien festlegt, definiert. Eine Ausnahme bilden die beiden italienischen Provinzen Trentino und Südtirol, die als Provinzen an der makroregionalen Strategie teilnehmen. Daneben verweist der Begriff Region auch auf die Alpenregion als Raum ohne administrative Grenzen.

22 Europäische Kommission, »Mitteilung der Kommission an das Europäische Parlament, den Rat, den Europäischen Wirtschafts- und Sozialausschuss und den Ausschuss der Regionen zur Strategie der Europäischen Union für den Ostseeraum«, $\operatorname{KOM}(2009)$ 248/3, Brüssel, 10. Juni 2009.

23 Alpenregionen, »Gemeinsame Erklärung anlässlich des Regionen-Gipfels zur Alpenstrategie am 12.03.2010 in Mittenwald « www.alpconv.org/de/organization/gro ups/WGMacroregionalstrategy/Documents/20120130_AlpengipfelDTweb.pdf (abgerufen am 23. Dezember 2017).

24 Konferenz der Alpenregionen, »Makroregionale Strategie für den Alpenraum. Initiativpapier der Alpenregionen" (Bad Ragaz, 29. Juni 2012) www.argealp.org/con tent/download/2521/14858/version/5/file/Initiativpapier_fin_DE.docx.pdf (abgerufen am 23. Dezember 2017).

25 Staaten und Regionen der Alpenregion, »Political Resolution towards a European Union Strategy for the Alpine Region « (Grenoble, 2013) http://ec.europa.eu/regio nal_policy/sources/cooperate/alpine/grenoble_131018_resolution_fin_sig_en.pdf (abgerufen am 31. Dezember 2017). 
mission Ende 2013 mit der Ausarbeitung der Strategie beauftragte. ${ }^{26}$ Die Alpenraumstrategie wurde schließlich im Juli 2015 von der Europäischen Kommission präsentiert ${ }^{27}$ und vom Europäischen Rat bestätigt.

An der Alpenraumstrategie nehmen die sieben Staaten Deutschland, Frankreich, Italien, Liechtenstein, Österreich, Schweiz und Slowenien sowie 48 Regionen teil. Für die Implementierung wurden drei neue Gremien - eine politische Generalversammlung, der technische Exekutivausschuss und neun Aktionsgruppen ${ }^{28}$ - geschaffen (vgl. Abbildung 1). Eine Generalversammlung bildet die politische Ebene. Politiker der teilnehmenden 48 Regionen und sieben Staaten verabschieden dort die grundsätzlichen Leitlinien der Zusammenarbeit. Die Europäische Kommission und die Alpenkonvention, ein zwischenstaatlicher Vertrag, der sich der nachhaltigen Entwicklung der Alpen verpflichtet hat, nehmen teil, verfügen aber über kein Stimmrecht. Eine jährlich wechselnde Präsidentschaft, die von Nationalstaaten oder Regionen übernommen wird, setzt Impulse und unterstützt den Prozess. Der Exekutivausschuss ist die operative Steuerungsebene. Er setzt sich aus sieben nationalstaatlichen Delegationen zusammen, die Beamte der nationalen und der regionalen Ebene der sieben Alpenstaaten umfassen. Daneben nehmen die Alpenkonvention und das Alpenraumprogramm, das EU-Kooperationsprogramm für den Alpenraum, als Beobachter teil. Die neun Aktionsgruppen stimmen Politiken ab und entwickeln konkrete Projekte. Sie setzen sich vor allem aus Vertretern der nationalen und regionalen Verwaltungen zusammen.

26 Europäischer Rat, »Schlussfolgerungen«, Tagung vom 19./20. Dezember 2013, Brüssel. http://data.consilium.europa.eu/doc/document/ST-217-2013-INIT/de/pdf (abgerufen am 13. April 2018).

27 Europäische Kommission, »Mitteilung der Kommission an das Europäische Parlament, den Rat, den Europäischen Wirtschafts- und Sozialausschuss und den Ausschuss der Regionen zu einer Strategie der Europäischen Union für den Alpenraum«, $\operatorname{COM}(2015) 366$ final, Brüssel, 28. Juli 2015; Europäische Kommission, "Commission Staff Working Document Accompanying the Communication from the Commission to the European Parliament, the Council, the European Economic and Social Committee and the Committee of the Regions Concerning the European Union Strategy for the Alpine Region, Action Plan«, $\operatorname{COM}(2015) 366$ final, Brüssel, 28. Juli 2015.

28 Vgl. Europäische Kommission, »Commission Staff Working Document« (Fn. 27), $47 \mathrm{ff}$. 
Abbildung 1: Gremien der Alpenraumstrategie

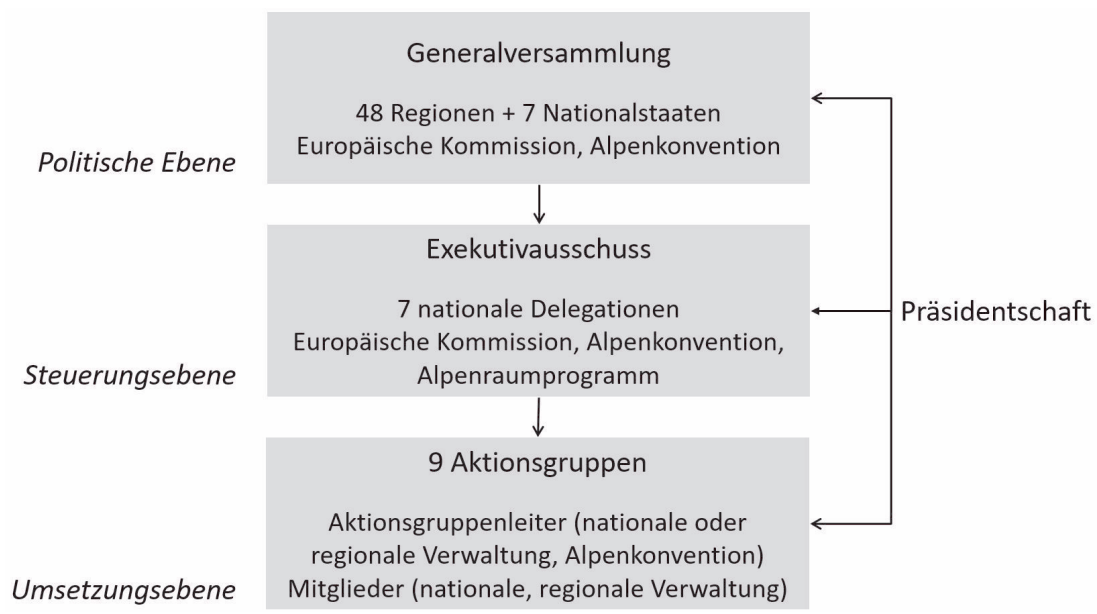

Der vorliegende Beitrag untersucht, inwiefern die Entscheidungs- und Implementationsstrukturen der Alpenraumstrategie den Postulaten demokratischer Legitimität entsprechen. Dabei werden unterschiedliche Demokratiemodelle in den Blick genommen, die territoriale und funktionale Repräsentation sowie direkte, partizipative und deliberative Teilhabe betonen. Die Fallstudie beruht auf einer umfangreichen Dokumentenanalyse, qualitativen Interviews und der teilnehmenden Beobachtung an Treffen und Konferenzen. In einem ersten Schritt wurde eine große Bandbreite von Strategiedokumenten, Positionspapieren, Protokollen und Korrespondenz analysiert, die von beteiligten subnationalen und nationalen Regierungen zur Verfügung gestellt und in einer Internetrecherche identifiziert wurden. In einem zweiten Schritt wurden zwischen Mai 2016 und September 201750 Interviews mit Vertretern der subnationalen und nationalen Verwaltungen, europäischer Institutionen, internationaler Organisationen sowie Nichtregierungsorganisationen in Belgien, Deutschland, Frankreich, Italien, der Schweiz, Liechtenstein und Österreich geführt. Zusätzliche Informationen zur Umsetzung demokratischer Normen wurden im Rahmen von Aktionsgruppentreffen und Konferenzen gesammelt. Die Daten wurden mit dem Computerprogramm MAXQDA 12 ausgewertet. Das nächste Kapitel beschäftigt sich zunächst mit der Alpenraumstrategie vor dem Hintergrund repräsentativer Demokratiemodelle. 


\section{Legitimität und Repräsentation}

\subsection{Territoriale Repräsentation}

Das Modell der territorialen Repräsentation dient im nationalstaatlichen Kontext als primärer Maßstab zur Bewertung demokratischer Legitimität. ${ }^{29}$ Bürger speisen dabei ihre Präferenzen nicht unmittelbar in den Entscheidungsprozess ein, sondern ermächtigen politische Vertreter, in ihrem Namen Entscheidungen zu treffen. Die Ermächtigung zur Vertretung erfolgt durch regelmäßig stattfindende Wahlen, die die Präferenzen eines territorialen Wahlkreises, etwa eines Nationalstaates oder einer Region, aggregieren. Damit garantiert das repräsentative Demokratiemodell formell gleiche Beteiligung durch Wahlen, schränkt zugleich aber darüber hinausgehende Partizipationsmöglichkeiten ein. Eine wichtige Rolle kommt Parteien zu, die Interessen und Wünsche der Bürger aggregieren und im politischen Prozess einbringen. Der Widerstreit politischer Ideen erfolgt in Parlamenten, die Kompromisse zwischen den verschiedenen parteipolitisch organisierten Interessen ermöglichen.

Die Legitimität von politischen Entscheidungen hängt damit davon $\mathrm{ab}$, dass durch Wahlen direkt legitimierte Vertreter an Entscheidungen mitwirken, die Interessen ihrer Wahlkreise einbringen und von diesen sanktioniert werden können. Auf der Input-Dimension beschäftigt sich repräsentative Demokratie vor allem mit der Qualität der Repräsentation, die verschiedene Interessen inkludieren und repräsentieren soll. ${ }^{30}$ Die Throughput-Dimension betont, dass gewählte Politiker, die Präferenzen in Wahlen aggregieren, gleichwertig an Entscheidungen mitwirken müssen. ${ }^{31}$ Zentral sind zudem institutionelle Kontrollmechanismen (checks und balances), die verhindern, dass eine Institution unbegrenzt Macht ausüben kann, und die dafür sorgen, dass auch Minderheiteninteressen im Entscheidungsprozess Berücksichtigung finden. ${ }^{32}$ Die Output-Dimension beschäftigt sich damit, inwiefern die Resultate mit politisch definierten Zielen übereinstimmen und den aggregierten Wünschen der Bürger entsprechen. Daneben muss gewährleistet sein, dass Bürger ihre Volksvertre-

29 Vgl. John Stuart Mill, Considerations on Representative Government (Cambridge University Press, 2010).

30 Bekkers und Edwards, »Legitimacy and Democracy« (Fn. 9), $64 \mathrm{f}$.

31 Berberich und Röber, »Demokratiedefizite europäischer Grenzregionen« (Fn. 8), 134.

32 Bekkers und Edwards, »Legitimacy and Democracy«(Fn. 9), 65 f. 
ter kontrollieren können. ${ }^{33}$ Dafür müssen sie über Informationen über getroffene Entscheidungen und Maßnahmen verfügen, um diese auch im Rahmen von Wahlen sanktionieren zu können.

Bei der normativen Bewertung von grenzüberschreitender Kooperation erweist sich das Modell territorialer Demokratie als besonders einflussreich. Da grenzüberschreitende Kooperation in Europa dem klassischen nationalstaatlichen Modell folgt, nach dem öffentliche Gebietskörperschaften in klar definierten territorialen Räumen möglichst viele verschiedene Themen umfassend regeln, ${ }^{34}$ wird auch der normative Demokratieanspruch häufig nach dem nationalstaatlichen Vorbild modelliert. Territoriale Repräsentation in grenzüberschreitender Kooperation kann dabei auf zwei Arten erfolgen. Eine erste Form von territorialer Repräsentation impliziert, dass alle Entscheidungsträger an ihre eigenen, nicht grenzüberschreitenden Wahlkreise gebunden bleiben. Legitim ist grenzüberschreitendes Handeln damit vor allem dann, wenn gewählte Politiker der regionalen sowie nationalstaatlichen Exekutive und der Parlamente substantiellen Einfluss auf Prioritäten, Themen und Resultate nehmen und über Wahlen sanktioniert werden können. ${ }^{35}$

Diese Form der indirekten Legitimität stößt im grenzüberschreitenden Kontext allerdings an einige Grenzen. So erfolgt in der Regel ex-ante keine formelle Autorisierung von Repräsentation. Da nationale, regionale und lokale Legislativen in grenzüberschreitender Kooperation eine geringe Rolle spielen, entscheidet die parlamentarische Debatte auch nicht darüber, was eingebracht wird. ${ }^{36}$ Politiker der Legislative, aber auch der Exekutive sind häufig wenig in Planungen und Entscheidungen eingebunden, ${ }^{37}$ während Beamte Entscheidungen auch zum Rahmen der Kooperation, etwa zur Auswahl der Teilnehmer oder zur thematischen Agenda,

33 Ebd., 59 und 66.

34 Joachim Blatter, "From sSpaces of Places to sSpaces of Flows?? Territorial and Functional Governance in Cross-Border Regions in Europe and North America", (2004) 28 International Journal of Urban and Regional Research 540.

35 Vgl. Eva Sørensen und Jacob Torfing, »The Democratic Anchorage of Governance Networks«, (2005) 28 Scandinavian Political Studies 195, 202; Hall, »Opportunities for Democracy« (Fn. 7).

36 Kratochwill und Fröhlich, »Legitimität und demokratische Potenziale« (Fn. 7), 68; Yannis Papadopoulos, "Accountability and Multi-Level Governance: More Accountability, Less Democracy?«, (2010) 33 West European Politics 1030, $1034 \mathrm{f}$.

37 Hall, »Opportunities for Democracy« (Fn. 7). 
treffen..$^{38}$ Grenzüberschreitende Kooperation bricht zudem parteipolitische Repräsentationsmuster auf, da Beamte und Politiker meist ihre territorialen Institutionen und nicht Parteien vertreten. ${ }^{39}$

Zugleich sind im grenzüberschreitenden Kontext Probleme und Lösungen, und damit Ziele und Aktivitäten politischer Akteure, häufig nicht im Vorhinein bekannt, sondern werden im Prozess definiert und verändert. ${ }^{40}$ Die Erteilung eines klaren Mandats im Vorhinein über Wahlen, und die nachträgliche Prüfung dieses Mandats, sind damit schwer möglich. ${ }^{41}$ Die Rückbindung der Akteure an klassische Muster der Repräsentation mindert ihren Handlungsspielraum und erschwert konsensorientiertes Verhandeln. ${ }^{42}$ Wenn Entscheidungstragende zunächst darauf warten müssen, dass eine offizielle Position der politischen Ebene, idealerweise in einem parlamentarischen Prozess, vorgegeben wird, kommt es zu langen Prozessen und Entscheidungsblockaden. Eine zu enge Bindung an den Wahlkreis unterminiert damit die Fähigkeit, verhandelte Vereinbarungen zu erreichen. ${ }^{43}$

Schließlich fehlen ex-post Möglichkeiten zur Zustimmung und Kontrolle von Repräsentation. ${ }^{44}$ Aufgrund der Komplexität der Prozesse, der vielen beteiligten Akteure und der Notwendigkeit von Kompromissen sind grenzüberschreitende Arenen häufig intransparent und informell organi-

38 Eva Sørensen, »Metagovernance: The Changing Role of Politicians in Processes of Democratic Governance", (2006) 36 The American Review of Public Administration 98; Papadopoulos, "Accountability and Multi-Level Governance« (Fn. 36), $1034 \mathrm{f}$.

39 Ian Bache und Jan Olsson, »Legitimacy through Partnership? EU Policy Diffusion in Britain and Sweden«, (2001) 24 Scandinavian Political Studies 215, $231 \mathrm{f}$.

40 Erik-Hans Klijn und Johannes Franciscus Maria Koppenjan, Managing Uncertainties in Networks: A Network Approach to Problem Solving and Decision Making (Routledge, 2004); Maarten Hajer, »A Frame in the Fields: Policymaking and the Reinvention of Politics«, in Maarten Hajer und Hendrik Wagenaar (Hrsg.), Deliberative Policy Analysis. Understanding Governance in the Network Society (Cambridge University Press, 2006).

41 Charles F. Sabel und Jonathan Zeitlin, »Learning from Difference: The New Architecture of Experimentalist Governance in the EU«, in Charles F. Sabel und Jonathan Zeitlin (Hrsg.), Experimentalist Governance in the European Union. Towards a New Architecture (Oxford University Press, 2010), 11.

42 Benz, »Postparlamentarische Demokratie?«(Fn. 14), 205 ff; Eva Sørensen, »Governance Networks as a Frame for Inter-Demoi Participation and Deliberation", (2012) 34 Administrative Theory \& Praxis 509, 524.

43 Berberich und Röber, »Demokratiedefizite europäischer Grenzregionen« (Fn. 8), 141.

44 Papadopoulos, »Accountability and Multi-Level Governance« (Fn. 36), 1037. 
siert. ${ }^{45}$ Es fällt damit schwer, Behauptungen der Repräsentation und den Beitrag der verschiedenen Akteure an Entscheidungen nachzuvollziehen, zu kontrollieren und zu sanktionieren. ${ }^{46}$ Grenzüberschreitende Kooperation ist bei Wahlen kaum Thema und fließt daher auch nicht in die Bewertung der Repräsentanten ein. Die teilnehmenden Beamten sind zudem nicht durch Wahlen sanktionierbar. Daneben werden formelle checks und balances ausgehebelt, um Blockaden im Mehrebenensystem angesichts vielfältiger Veto-Spieler zu vermeiden. So werden der Einbezug von Parlamenten oder der Bezug auf formelle Verfahren häufig als bremsend erlebt und daher umgangen. ${ }^{47}$

Die Alpenraumstrategie stellt vor diesem Hintergrund eine demokratische Verbesserung dar. Da es sich um eine Strategie handelt, die bestehende Aktivitäten im grenzüberschreitenden Raum zusammenführen und hinter einer strategischen Zielsetzung vereinen soll, sieht sie auch eine politische Verankerung in den teilnehmenden Staaten und Regionen vor. Direkt in ihren territorialen Wahlkreisen gewählte Politiker spielen daher eine wichtige Rolle. Bereits in der Erarbeitungsphase waren Politiker aktiv eingebunden, gaben politische Zielsetzungen vor und verabschiedeten erste inhaltliche Papiere. Die Bestätigung der Strategie durch den Europäischen Rat, und damit durch die Staats- und Regierungschefs aller EU-Mitgliedstaaten, gibt der Strategie eine in den territorialen Nationalstaaten verankerte Legitimation.

Die Umsetzung der makroregionalen Strategie sieht regelmäßige Treffen der politischen Ebene vor, die im Rahmen der Generalversammlung grenzüberschreitende Themen debattieren und Grundsatzentscheidungen treffen soll. Daneben gibt es zu bestimmten Themen auch Fachministertreffen. Allerdings nehmen die politischen Vertreter vieler Nationalstaaten und Regionen nicht an den Treffen teil. Viele Politiker beschäftigen sich häufig aus Zeitmangel oder mangelndem Interesse nur peripher mit den Inhalten der Strategie. Politiker in der Generalversammlung vertreten zudem weniger parteipolitische Positionen als ihre territorialen Institutionen. Dies birgt die Gefahr, dass parteipolitische Differenz im Namen

45 Michael T. Greven, »The Informalization of Transnational Governance: A Threat to Democratic Government «, in Edgar Grande und Louis W. Pauly (Hrsg.), Complex Sovereignty: Reconstituting Political Authority in the Twenty-First Century (University of Toronto Press, 2005).

46 Ebd.; Sørensen, »Governance Networks «(Fn. 42), 517 f.

47 Arthur Benz, »Path-Dependent Institutions and Strategi c Veto Players: National Parliaments in the European Union«, (2004) 27 West European Politics 875. 
territorialer Wahlkreise eingeebnet wird. ${ }^{48}$ Repräsentation ist zudem auf die nationale und regionale Ebene beschränkt, während Vertreter lokaler Gebietskörperschaften nicht an der Generalversammlung teilnehmen.

Politische Treffen enden in der Regel mit einer politischen Deklaration, in der die teilnehmenden territorialen Vertreter grundsätzliche Leitlinien vorgeben. ${ }^{49}$ Allerdings bleiben diese Grundsatzbeschlüsse häufig vage und behandeln wenig kontroverse Themen. Das Konsensprinzip macht es schwer, Entscheidungen zu treffen, die negative Konsequenzen für einzelne Teilnehmer mit sich bringen. ${ }^{50}$ Eine Politisierung der Debatte wird zudem durch die funktionale Zielsetzung, die sich an »objektiven" Problemen des Alpenraumes orientiert und damit Interessenkonflikte diskursiv einebnet, unterminiert. ${ }^{51}$ Da nur die Deklarationen, nicht allerdings Protokolle oder weiterführende Informationen auf der Homepage zur Verfügung gestellt werden und die Sitzungen nicht öffentlich sind, ist es schwierig für die Bevölkerung, Verantwortlichkeiten zuzuweisen und Verhalten zu sanktionieren.

Die Parlamente haben eine formell schwache Stellung in der Alpenraumstrategie, konnten sich aber fallweise durch einzelne engagierte Politiker einbringen. Teilweise wurde die Alpenraumstrategie in den Regional- und Nationalparlamenten thematisiert. ${ }^{52}$ Hervorzuheben ist auch die Behandlung des Themas im Dreierlandtag des EVTZ Tirol-Südtirol-Trenti-

48 Hall, »Opportunities for Democracy« (Fn. 7), 430 f; Bache und Olsson, »Legitimacy through Partnership? (Fn. 39), $231 \mathrm{f}$.

49 Vgl. »Brdo Joint Statement on the European Union Strategy for the Alpine Region (EUSALP)«(Brdo, 25. Januar 2016) http://eusalp.digital-distillery.net/sites/de fault/files/uploads/event/318/attachments/eusalpjointstatement25012016.pdf (abgerufen am 31. Dezember 2017); "Joint Declaration on the EU Strategy for the Alpine Region (EUSALP)« (Rottnach-Egern, 13. Februar 2017) www.alpine-regio n.eu/sites/default/files/uploads/news/478/ attachments/17_02_13_joint_declaration_rottach-egern_en.pdf (abgerufen am 31. Dezember 2017); »Joint Declaration on the EU Strategy for the Alpine Region (EUSALP) (München, 23. November 2017) www.bayern.de/wp-content//uploads/2017/12/17_11_23-joint-declaration-m unich-en-final-change.pdf (abgerufen am 31. Dezember 2017).

50 Berberich und Röber, »Demokratiedefizite europäischer Grenzregionen« (Fn. 8), $138 \mathrm{f}$.

51 Hall, »Opportunities for Democracy« (Fn. 7), 429 f.

52 Vgl. etwa Deutscher Bundesrat, »Beschluss des Bundesrates: Mitteilung der Kommission an das Europäische Parlament, den Rat, den Europäischen Wirtschaftsund Sozialausschuss und den Ausschuss der Regionen zu einer Strategie der Europäischen Union für den Alpenraum, $\operatorname{COM(2015)~} 366$ final«, 329/15, 25. September 2015. 
no. ${ }^{53}$ Das Europäische Parlament hat zudem zwei Resolutionen zur Alpenraumstrategie verabschiedet ${ }^{54}$ und der makroregionalen Strategie Gelder im Rahmen des Alpine Region Preparatory Action Fund (ARPAF) zugewiesen. Eine eigene interregionale Gruppe im Europäischen Parlament befasst sich mit der makroregionalen Strategie. Es gibt damit mehrere parlamentarische Arenen, in denen die Alpenraumstrategie debattiert wird. Wichtigste Verbindung zwischen den Parlamenten und der makroregionalen Strategie sind allerdings weniger formelle Verfahren als informelle Netzwerke ${ }^{55}$ sowie einzelne aktive Politiker, etwa die Präsidenten einiger Regionalparlamente oder Abgeordnete des Europäischen Parlaments, die an der Generalversammlung als Vertreter ihrer Wahlkreise teilnehmen.

Eingeschränkt ist die repräsentative Dimension schließlich dadurch, dass alle operativen Entscheidungen im Exekutivausschuss und in den Aktionsgruppen fallen. Die Beamten sind, sofern die politische Ebene diese vorgibt, politischen Zielsetzungen verpflichtet und stehen im Austausch mit Politikern. Daneben benötigen die Vertreter im Exekutivausschuss sowie in den Aktionsgruppen ein klares Mandat ihrer Region oder ihres Nationalstaates, um an Entscheidungen mitwirken zu können. ${ }^{56} \mathrm{Da}$ der grenzüberschreitende Kontext es allerdings nicht zulässt, alle Möglichkeiten im Vorfeld zu regeln, kommt den Beamten auch ein Ermessensspielraum zu. Zudem haben sie gegenüber den Politikern, die sich meist nur fallweise mit der Alpenraumstrategie befassen, einen Informationsvorsprung. Die Europäische Kommission erarbeitet die Strategiedokumente, bereitet viele Vorschläge vor und koordiniert den generellen Prozess. Sie

53 Gemeinsame Sitzung des Südtiroler, Tiroler und Trentiner Landtags, Beschluss Nr. 1 betreffend Makroregionale EU-Strategie für den Alpenraum, Schwaz, 28. Oktober 2014.

54 Europäisches Parlament, »Entschließung des Europäischen Parlaments vom 23. Mai 2013 zu einer makroregionalen Strategie für die Alpen (2013/2549 $(\mathrm{RSP})) \ll$; Europäisches Parlament, »Entschließung des Europäischen Parlaments vom 13. September $2016 \mathrm{zu}$ einer EU-Strategie für den Alpenraum $(2015 / 2324(\mathrm{INI})) \ll$.

55 Benz, »Path-Dependent Institutions« (Fn. 47).

56 Strategie der Europäischen Union für den Alpenraum, »Rules of Procedure for the Executive Board of the EU strategy for the Alpine Region « (2016) www.alpine -region.eu/sites/default/files/uploads/page/24/attachments/eusalp ebrop24102016.pdf (abgerufen am 26. Dezember 2017); Strategie der Europäischen Union für den Alpenraum, »Rules of Procedure for the Action Groups of the EU strategy for the Alpine Region« (2016) www.alpine-region.eu/sites/default/ files/uploads/page/24/attachments/eusalpagsrop24102016.pdf (abgerufen am 26. Dezember 2017). 
hat damit eine wesentliche Agenda-Setting-Rolle, die die Verbindung zu demokratisch legitimierter Repräsentation schwächt.

Die Alpenraumstrategie durchbricht damit das Modell territorialer Repräsentation in vielfacher Hinsicht. Parlamente, Parteien und Politiker treten hinter Netzwerke von Beamten zurück. Zugleich stärkt die Strategie die Repräsentation territorialer Interessen im Vergleich zu anderen grenzüberschreitenden Strukturen, da sie eine aktive Einbindung der politischen Ebene und die Formalisierung von Entscheidungsprozessen vorsieht. Das Modell territorialer Repräsentation stößt in der grenzüberschreitenden Sphäre allerdings auch an seine Grenzen. Da es keine Mechanismen der Repräsentation von grenzüberschreitenden Räumen gibt, sondern jeder nur seinen eigenen Wahlkreis vertritt, kann niemand den Anspruch erheben, für den Alpenraum als grenzüberschreitende Region zu sprechen. Damit kann auch keiner der beteiligten Akteure Forderungen gegenüber der EU oder den Nationalstaaten im Namen der grenzüberschreitenden Region stellen. ${ }^{57}$ Der Wunsch, Kompetenz und Legitimität an die eigene nationalstaatliche oder regionale Kompetenzsphäre zu binden, steht damit im Spannungsverhältnis zum Anspruch, Probleme und Potentiale zu adressieren, die die grenzüberschreitende Sphäre als Ganzes betreffen..$^{58}$

Eine zweite Möglichkeit, grenzüberschreitende Kooperation nach dem Modell territorialer Repräsentation zu legitimieren, besteht daher in der Schaffung grenzüberschreitender Repräsentation. Der Transfer territorialer Repräsentation auf die grenzüberschreitende Ebene erfordert grenzüberschreitende Wahlen. Die teilnehmenden territorialen Einheiten müssten Teile ihrer Souveränität an ein grenzüberschreitendes Parlament abgeben. ${ }^{59}$ Allerdings kann, selbst wenn die teilnehmenden Regierungen bereit dazu sind, Souveränität abzugeben, die Einrichtung einer parlamentarischen Versammlung zu Überlappungen und damit zu Konflikten um Verantwortlichkeiten und Kompetenzen führen. ${ }^{60}$ Da die teilnehmenden Staaten und Regionen häufig sehr unterschiedlich groß sind, ist zudem

57 Kramsch und Mamadouh, »Crossing Borders« (Fn. 7), 43.

58 Olivier Kramsch, »Navigating the Spaces of Kantian Reason: Notes on Cosmopolitical Governance within the Cross-Border Euregios of the European Union«, (2001) 6 Geopolitics 27, $41 \mathrm{f}$.

59 Kratochwill und Fröhlich, »Legitimität und demokratische Potenziale« (Fn.7), 72.

60 Hall, »Opportunities for Democracy« (Fn. 7), 433. 
die Gefahr groß, dass nicht jede Stimme dasselbe Gewicht erhält. ${ }^{61}$ Daneben wird von einigen Autoren angemerkt, dass ein grenzüberschreitendes Parlament, das politische Mehrheitsentscheidungen fällt, ein einheitliches Demos, das sich auch als solches begreift und an grenzüberschreitenden öffentlichen Diskursen teilnimmt, voraussetzt. ${ }^{62}$ Andere Autoren merken an, dass Institutionen selbst Diskurse, Identität und ein grenzüberschreitendes Demos schaffen können. ${ }^{63}$ Zurzeit gibt es in einigen grenzüberschreitenden Kooperationen Parlamente, ${ }^{64}$ die allerdings nicht direkt gewählt werden, sondern sich aus Delegierten der teilnehmenden Staaten und Gebietskörperschaften zusammensetzen. Damit stärken sie die Kontrollfunktion der nationalen und regionalen Parlamente, führen aber nicht zur Schaffung eines grenzüberschreitenden Wahlkreises. ${ }^{65}$ In der Alpenraumstrategie gibt es bislang kein grenzüberschreitendes Parlament, das Interessen des Alpenraumes repräsentiert.

In Summe entstehen beim Versuch, das Modell territorialer Repräsentation auf die grenzüberschreitende Sphäre zu übertragen, viele Schwierigkeiten. Die Alpenraumstrategie kann weder den notwendigen direkten Bezug zu bestehenden territorialen Wahlkreisen noch die Schaffung eines neuen, demokratisch legitimierten grenzüberschreitenden Wahlkreises herstellen. Der Beitrag wendet sich daher einem zweiten Mechanismus von Repräsentation, der funktionalen Repräsentation, zu.

61 Robert Dahl, „Can International Institutions Be Democratic? A Skeptic’s View«, in Ian Shapiro and Casiano Hacker-Cordón (Hrsg.), Democracy's Edges (Cambridge University Press, 1999), 31.

62 Ebd.; Fritz W. Scharpf, Governing in Europe: Effective and Democratic? (Oxford University Press, 1999); Kratochwill und Fröhlich, »Legitimität und demokratische Potenziale« (Fn. 7), 72.

63 Lars-Erik Cederman, "Nationalism and Bounded Integration: What it Would Take to Construct a European Demos«, (2001) 7 European Journal of International Relations 139; Julia Boman und Eiki Berg, »Identity and Institutions Shaping Cross-Border Co-Operation at the Margins of the European Union", (2007) 17 Regional \& Federal Studies 195.

64 Beispiele sind die Baltic Sea Parliamentary Conference im Ostseeraum, der Dreierlandtag des EVTZ Tirol-Südtirol-Trentino oder die Internationale Parlamentarische Bodensee-Konferenz in der Bodensee-Region.

65 Christopher Lord, "Democracy and the European Union: Matching Means to Standards«, (2006) 13 Democratization 668, 671. 


\subsection{Funktionale Repräsentation}

Funktionale Repräsentation beruht darauf, dass die Bevölkerung nicht von gewählten Politikern, sondern von Interessengruppen vertreten wird. Diese Repräsentation wird vor allem im Modell pluralistischer Demokratie ${ }^{66}$ betont, das der institutionellen Politik eine Rolle als neutrale Vermittlerin zuweist. Politische Entscheidungen beruhen nicht auf dem Wettstreit von Parteien in Parlamenten, sondern auf dem Wettbewerb, den Verhandlungen und der Koalitionsbildung zwischen Interessengruppen. Politische Macht ist damit unter vielfältigen Interessengruppen verteilt. Entscheidungen sind weniger durch territoriale Grenzen und formalisierte Regeln limitiert, und die Interessenaggregation erfolgt nicht nach territorialen, sondern nach funktionalen Kriterien. Jeder Bürger kann sich frei einer Interessengruppe anschließen und an den Positionen der Interessengruppe mitwirken.

Die Legitimität politischer Entscheidungen hängt davon ab, ob vielfältige Interessengruppen existieren, gleiche Möglichkeiten zur Teilnahme haben und von ihren Mitgliedern sanktioniert werden können. Auf der Inputdimension aggregieren Interessengruppen die Präferenzen ihrer Mitglieder und eröffnen damit Partizipationsmöglichkeiten. ${ }^{67}$ Der Prozess der Interessenaggregation ist allerdings davon abhängig, inwiefern alle Mitglieder auch gleiche Möglichkeiten haben, die Positionen der Interessengruppen mitzubestimmen. Die Throughput-Dimension betont, dass es einen freien Wettbewerb der verschiedenen Interessengruppen geben muss, der frei von asymmetrischen Machtverhältnissen ist. ${ }^{68}$ Ein unterschiedlicher Zugang der Interessengruppen zu Ressourcen und Entscheidungsarenen unterminiert die Legitimität von Entscheidungen. Auf der Output-Seite soll der Einbezug von Interessengruppen Resultate ermöglichen, die mit den Wünschen der Bürger korrespondieren. Zugleich erfolgt die Verantwortlichkeit über die Interessengruppen, die von ihren Mitgliedern für Entscheidungen verantwortlich gehalten werden. ${ }^{69}$

66 Claude J. Burtenshaw, »The Political Theory of Pluralist Democracy«, (1968) 21 The Western Political Quarterly 577; Robert Dahl, Pluralist Democracy in the United States: Conflict and Consent (Rand McNally, 1968); Robert Dahl, Democracy and its Critics (Yale University Press, 1989); Ernst Fraenkel, Deutschland und die westlichen Demokratien (Mit einem Nachwort über Leben und Werk Ernst Fraenkels, hrsg. von Alexander Brünneck, Suhrkamp, 1991).

67 Bekkers und Edwards, »Legitimacy and Democracy« (Fn. 9), 69.

68 Fraenkel, Deutschland und die westlichen Demokratien (Fn. 66), 358.

69 Bekkers und Edwards, »Legitimacy and Democracy« (Fn. 9), 69. 
Funktionale Repräsentation im grenzüberschreitenden Kontext impliziert, dass verschiedene Interessengruppen frei an der Kooperation und an Entscheidungen mitwirken können. Diese Interessengruppen können auch grenzüberschreitend organisiert sein. Damit erlaubt es das Modell funktionaler Repräsentation, Legitimität auch in wenig formalisierten und territorial nicht einheitlich repräsentierten grenzüberschreitenden Räumen herzustellen. Zugleich trägt es dem Umstand Rechnung, dass asymmetrische Ressourcen und ein unterschiedliches Bewusstsein eine Beteiligung aller Bürger an Entscheidungen erschweren. Die Involvierung von Interessengruppen erscheint vor diesem Hintergrund als eine Möglichkeit, den Einfluss von Bürgern, die in den Interessengruppen direkt Einfluss auf Positionen nehmen können, auf verschiedene Politikbereiche zu stärken. ${ }^{70}$ Interessengruppen fungieren dabei als intermediäre Organisationen, die zwischen dem öffentlichen Raum grenzüberschreitender Kooperation und den Bürgern vermitteln. Legitimität, die sich ausschließlich auf funktionale Repräsentation stützt, steht allerdings vor dem Problem, dass Interessengruppen nur ihren Spendern und Mitgliedern gegenüber verantwortlich sind, nicht aber der generellen Öffentlichkeit oder den Gemeinschaften, die von ihren Aktionen betroffen sind. ${ }^{71}$ Daher werden in der Praxis meist Formen der territorialen und der funktionalen Repräsentation miteinander verbunden.

Die Beteiligung von Interessengruppen an Entscheidungen ist in der grenzüberschreitenden europäischen Kooperation allerdings bislang wenig ausgeprägt. ${ }^{72}$ Der Einbezug in der Alpenraumstrategie ist auf wenige Bereiche limitiert und wenig strukturiert. Im Erarbeitungsprozess konnten sich Interessengruppen teilweise in Untergruppen einbringen. Die Auswahl der Interessenvertreter, die einbezogen wurden, orientierte sich allerdings nicht an objektiven Kriterien, sondern an Netzwerken, Kontakten und Ressourcen sowie dem aktiv artikulierten Wunsch, teilzunehmen. Insbesondere Interessengruppen, die bereits auf der Ebene des Alpenraumes organisiert waren oder Kontakt zu den Entscheidungsträgern hatten, konnten dabei Interessen einbringen. Daneben wurden Interessengruppen teilweise in den einzelnen Regionen und Staaten im Rahmen von Koordi-

70 Matt Wood, »Between Technocracy and Democracy: How Stakeholder Engagement might be a Solution to the EU's Legitimacy Problems", in Adam Hug (Hrsg.), Europe and the People: Examining the EU's Democratic Legitimacy (Foreign Policy Centre, 2016), 16 ff.

71 Fraenkel, Deutschland und die westlichen Demokratien (Fn. 66), 358; Papadopoulos, »Accountability and Multi-Level Governance« (Fn. 36), 1037.

72 Joachim Blatter, »From >Spaces of Place to >Spaces of Flows ?? $540 \mathrm{f}$. 
nationsplattformen oder punktuellen Veranstaltungen einbezogen. Eine weitere Möglichkeit, funktionale Interessen beizutragen, bot die öffentliche Konsultation der Europäischen Kommission, die einen Online-Fragebogen zur Partizipation bereitstellte. ${ }^{73}$ Allerdings bleibt hier offen, inwiefern Beiträge der Interessengruppen in Entscheidungen einflossen. Ein freier Wettbewerb der Interessengruppen fand daher im Erarbeitungsprozess nicht statt.

In den Entscheidungsgremien der Alpenraumstrategie nehmen keine Interessengruppen teil. Da eine Mitwirkung an Entscheidungen in den Aktionsgruppen ein Mandat eines Nationalstaates oder einer Region voraussetzt, können Interessengruppen sich auch hier nicht an Entscheidungen beteiligen. Die in den Gremien vertretenen öffentlichen Gebietskörperschaften ziehen dabei eine klare Linie zwischen territorialer und funktionaler Repräsentation:

Das ist diese Decision Making Capacity, "nominated by the respective $\mathrm{Na}$ tional Coordinator «, das heißt, der hat dann sozusagen das Mandat [...] und ist sozusagen in Rückkopplung, weil da soll gerechtfertigt werden, dass natürlich ein breiteres Interesse vertreten wird und nicht nur ein spezifisches. Weil wenn ich eine NGO [Non-governmental organisation, Nichtregierungsorganisation] zum Beispiel habe, die hat ein bestimmtes, enges Interesse, das sie vertritt. ${ }^{74}$

Es ist klar, dass die organisierte Zivilgesellschaft nicht genauso an Entscheidungen beteiligt werden kann wie die politischen Autoritäten. Das ist normal. Man ist in einem System, in dem die politischen Autoritäten die Legitimität der Wahlen besitzen, und daher ist es auch ganz normal, dass die politischen Autoritäten die letztgültigen Entscheidungen treffen. ${ }^{75}$

Den Aktionsgruppen kommt allerdings beim Einbezug von Interessengruppen ein bestimmter Ermessensspielraum zu. Einige Aktionsgruppen beziehen sie aktiv mit ein und ermöglichen ihre Teilnahme an Diskussionen und Projekten, während andere eine Teilnahme ausschließen. Objektive Kriterien, nach denen Interessenvertreter ausgewählt werden, fehlen allerdings. Interessengruppen werden daneben weiterhin über nationale

73 Europäische Kommission, »Public Consultation - Summary Report« (2014) http:/ /ec.europa.eu/regional_policy/sources/consultation/eusalp/pdf/summary_report.p df (abgerufen am 31. Dezember 2017).

74 Interview mit einem Beamten der Europäischen Kommission (Brüssel, 4. Oktober 2016).

75 Interview mit einem Beamten der französischen Behörde CGET (Commissariat général à l'égalité des territoires) (Paris, 28. November 2016), [übersetzt aus dem Französischen]. 
und regionale Koordinationsformate sowie Workshops und Veranstaltungen miteinbezogen. Ein weiterer Mechanismus, um die Beiträge der Interessengruppen zu sammeln, ist eine Wissensplattform, die im Internet Möglichkeiten zur Information, Vernetzung und Kontaktaufnahme bieten soll. Die verschiedenen Mechanismen sehen allerdings zumeist keine Beteiligung der Interessengruppen an Entscheidungen vor, sondern dienen vor allem der Sammlung von Expertise.

Ein stärkerer Einbezug von Interessengruppen in Entscheidungen könnte Partizipationsmöglichkeiten und damit die Legitimität erhöhen, ohne das Entscheidungsfeld für die breite Bevölkerung zu öffnen. Dafür wären allerdings objektive Kriterien für die Auswahl der Interessengruppen und eine breit angelegte Kommunikation und Mobilisierung notwendig. Wenn öffentliche Institutionen als Gatekeeper fungieren, müssen sie ihre Entscheidungen gut begründen können. Fehlen institutionalisierte Kanäle, ist der Einfluss der Interessengruppen stark von den Ressourcen, dem Organisationsgrad und der Fähigkeit, konsensfähige Positionen zu artikulieren und über verschiedene Kanäle zu kommunizieren, abhängig. Es besteht daher die Gefahr, dass Organisationen, die bereits grenzüberschreitend organisiert sind oder sich auf umfangreiche Ressourcen stützen können, Partizipationsprozesse monopolisieren. ${ }^{76}$ Funktionale Repräsentation, die Kriterien demokratischer Legitimität entspricht, muss daher Kapazitäten auch unter schwächeren Gruppen aufbauen. Einige Aktionsgruppen bieten mit der Refundierung der Reisekosten für Vertreter von Nichtregierungsorganisationen bereits Ansätze zur Stärkung funktionaler Repräsentativität.

Schließlich trägt der Einbezug von Interessengruppen nur dann zu mehr Legitimität bei, wenn es für die Mitglieder auch möglich ist, Verantwortlichkeit zuzuweisen und zu sanktionieren. Viele Interessengruppen verfügen über wenige demokratische Strukturen und bieten kaum Möglichkeiten zur Überprüfung von Entscheidungsprozessen. ${ }^{77}$ Eine enge Bindung an die eigene Mitgliederbasis, wie auch die Bindung an territoriale Wahlkreise im Modell territorialer Repräsentation, erschweren wiederum die Konsenssuche in Verhandlungen. ${ }^{78}$

76 Peter Bachrach und Morton S. Baratz, »Two Faces of Power«, (1962) 56 The American Political Science Review 947; Karin Bäckstrand, »Democratizing Global Environmental Governance? Stakeholder Democracy after the World Summit on Sustainable Development«, (2006) 12 European Journal of International Relations 467, 489.

77 Papadopoulos, »Accountability and Multi-Level Governance«(Fn. 36), $1037 \mathrm{f}$.

78 Benz, »Postparlamentarische Demokratie?« (Fn. 14), 209 ff. 
Repräsentative Mechanismen stoßen im grenzüberschreitenden Kontext an ihre Grenzen. Territoriale Repräsentation endet zumeist an den Grenzen der eigenen territorialen Einheit und lässt sich schwer auf die grenzüberschreitende Sphäre übertragen. Funktionale Repräsentation wird durch Ressourcenasymmetrien und Netzwerke geprägt. Um die demokratische Legitimität von grenzüberschreitender Kooperation zu erhöhen, können daher Modelle, die die direkte Beteiligung der Bevölkerung an Entscheidungs- und Implementierungsprozessen betonen, eine wertvolle Ergänzung darstellen. Das folgende Kapitel beschäftigt sich mit drei Modellen - direkter Demokratie, partizipativer Demokratie und deliberativer Demokratie -, die Teilhabe in den Mittelpunkt stellen.

\section{Legitimität und Teilhabe}

\subsection{Direkte Demokratie}

Das Modell der direkten Demokratie sieht vor, dass Wünsche und Interessen der Bürger direkt in Entscheidungen übertragen werden. Dies geschieht in der Regel über Referenden und Initiativverfahren, die verbindlich sind und die Meinung der Bürger in den politischen Prozess einspeisen. ${ }^{79}$ Der Kreis der zur Beteiligung Berechtigten wird in der Praxis meist territorial definiert, kann aber auch funktional, also nach bestimmten Sachthemen, abgegrenzt werden.

Auf der Input-Dimension steht damit die direkte und gleichberechtigte Beteiligung der Bürger im Vordergrund. Partizipation ist allerdings, in Bezug auf die Throughput-Dimension, zumeist an eine einfache Ablehnung oder Zustimmung zu bestimmten Initiativen gebunden. ${ }^{80}$ Die Gestaltungsdimension von Partizipation fällt damit gering aus. Die Möglichkeit, selbst Themen auf die Agenda zu setzen, gibt der Bürgerpartizipation eine stärker gestalterische Wirkung. ${ }^{81}$ Die Output-Dimension betont, dass Resultate den Wünschen der Bürger direkter entsprechen, weil sie direkt in Entscheidungsprozesse einbezogen werden. ${ }^{82}$ Ihre Kontrollmöglichkeiten

79 Lawrence LeDuc, The Politics of Direct Democracy: Referendums in Global Perspective (Broadview, 2003).

80 Bekkers und Edwards, »Legitimacy and Democracy« (Fn. 9), 67.

81 Oli Henman, »Europe by the People: How Could the EU's Structures Be More Directly Accountable to its Citizens?", in Hug (Hrsg.), Europe and the People (Fn. 70), 27.

82 Bekkers und Edwards, »Legitimacy and Democracy« (Fn. 9), 67. 
hängen allerdings auch davon ab, inwiefern sie bereits getroffene Entscheidungen etwa durch Referenden aufheben können.

Direkte Demokratie in grenzüberschreitender Kooperation würde sich in grenzüberschreitenden Abstimmungen zu bestimmten Sachthemen, ${ }^{83}$ aber auch in der Möglichkeit von Bürgern, bestimmte Themen auf die politische Agenda zu setzen, niederschlagen. Die Einführung direktdemokratischer Instrumente in grenzüberschreitender Kooperation könnte insbesondere die Input-Dimension von Legitimität stärken. Die Bürger würden motiviert, sich mit bestimmten Sachfragen im grenzüberschreitenden Kontext zu befassen. Damit könnte es auch zu einer stärkeren grenzüberschreitenden Bewusstseinsbildung kommen. In der Praxis ist Beteiligung allerdings durch die ungleiche Verfügbarkeit von Informationen, die Motivation der Bürger, sich zu beteiligen, oder die Bedeutung von Bildung für Partizipation beschränkt. ${ }^{84}$

In der makroregionalen Alpenraumstrategie gibt es bislang keine Möglichkeiten direkter Demokratie, da Referenden oder Initiativrechte auf die Regionen und Staaten beschränkt bleiben. Eine Möglichkeit wären allenfalls in allen Staaten und Regionen parallel stattfindende Plebiszite, deren Wirkung allerdings von den jeweiligen einzelstaatlichen Vorschriften abhängen würde. ${ }^{85}$ Direkte Demokratie setzt zudem die Schaffung transnationaler Arenen voraus, in denen öffentlich über Entscheidungen informiert und diskutiert werden kann. Dafür sind auch Medien notwendig, die Themen transnational debattieren und nicht in nationalstaatliche Einzelsphären herunterbrechen. Die transnationale Debatte von Sachfragen erscheint vor dem Hintergrund des großen geographischen Perimeters des Alpenraumes äußerst schwierig. Die Gefahr, dass die Debatte in viele Teilöffentlichkeiten zerfällt, ist groß. Abstimmungen würden damit eher mit der eigenen nationalstaatlichen oder regionalen Politik verbunden werden, während die grenzüberschreitende Thematik in den Hintergrund treten könnte. Die Einrichtung grenzüberschreitender Volksabstimmungen könnte diese Öffentlichkeit allerdings auch anregen und damit Raum für transnationale Debatten schaffen. Da direkte Demokratie Sachfragen

83 Kratochwill und Fröhlich, »Legitimität und demokratische Potenziale« (Fn. 7), 72.

84 Ian Budge, The New Challenge of Direct Democracy (Polity Press, 1996).

85 Maria Bertel, Teresa Sanader und Christoph Schramek, »Direkte Demokratie und Bürgerbeteiligung in der Europaregion - Ausgewählte Aspekte« (FöderalismusBlog, 7. Juli 2015) www.foederalismus.at/blog/direkte-demokratie-und-buergerbet eiligung-in-der-europaregion---ausgewaehlte-aspekte_60.php (abgerufen am 30. Dezember 2017). 
auf die beiden Optionen Zustimmung oder Ablehnung zuspitzt, integriert sie in geringerem Maße unterschiedliche Perspektiven und Hintergründe, die den grenzüberschreitenden Kontext prägen. Ein Modell, das dieser Komplexität stärker Rechnung trägt, ist das Modell partizipativer Demokratie.

\subsection{Partizipative Demokratie}

Eine möglichst breite Partizipation nicht nur im Rahmen von Abstimmungen, sondern im gesamten Entscheidungs- und Implementierungsprozess postuliert das Modell partizipativer Demokratie. ${ }^{86}$ Partizipation wird dabei weniger territorial als vielmehr funktional definiert. Alle von bestimmten Sachthemen Betroffenen sollen an Entscheidungen mitwirken können. Damit betont partizipative Demokratie vor allem die Input- und die Throughput-Dimension von Legitimität. Auf der Input-Seite stellt sich primär die Frage, inwiefern organisierte Interessen und Bürger direkt ihre Anliegen einbringen können und welche Möglichkeiten sie haben, eigene Themen auf der politischen Agenda zu platzieren. Auf der ThroughputDimension wird weniger die Aggregation von Präferenzen als vielmehr die Integration möglichst vieler verschiedener Interessen betont. Zudem geht es um die Vermeidung von Dominanz und Asymmetrie. Auf der Output-Dimension sollen Entscheidungen den eingebrachten Interessen entsprechen und Möglichkeiten dazu bieten, dass die Bürger ungewollte Resultate auch bewerten und sanktionieren können.

Da grenzüberschreitende Kooperation vielfältige Hintergründe und unterschiedliche Problemdefinitionen umfasst, kann Partizipation ein wertvolles Mittel sein, um die verschiedenen Perspektiven zu integrieren. Europäische grenzüberschreitende Kooperation bietet allerdings bislang wenige Möglichkeiten für partizipative Prozesse, die Beteiligung umfassend verankern. In der Alpenraumstrategie spielt direkte Teilhabe eine geringe Rolle. In den Erarbeitungsprozess wurden Bürger nicht direkt einbezogen. Vereinzelt fanden öffentliche Veranstaltungen in den teilnehmenden Staaten und Regionen statt, die allerdings eher der Information als der Partizipation an Entscheidungsprozessen dienten. Die breiteste Partizipationsmöglichkeit bot die öffentliche Konsultation der Europäischen Kommission.

86 Vgl. Carole Pateman, Participation and Democratic Theory (Cambridge University Press, 1970); Peter Bachrach und Aryeh Botwinick, Power and Empowerment: A Radical Theory of Participatory Democracy (Temple University Press, 1992). 
Über einen Online-Fragebogen konnten alle Interessierten Anliegen an die Alpenraumstrategie übermitteln. Grundlage der Konsultation war ein Konsultationspapier, das über die Strategie informierte und erste thematische Pfeiler festlegte. Voraussetzung für die Teilnahme war allerdings, dass die Bürger auch über ihre Möglichkeiten informiert waren. Die meisten Teilnehmer stammten daher auch von öffentlichen Gebietskörperschaften oder Interessengruppen. Zudem fehlte es an Kriterien, wie verschiedene Beiträge bewertet wurden und wie sie in die Entscheidungen einflossen. ${ }^{87}$ Es bleibt daher unklar, inwiefern die Strategie und der Aktionsplan die Konsultation widerspiegeln.

Bei der Umsetzung der Strategie bleiben sämtliche Entscheidungsgremien auf öffentliche Akteure beschränkt. Bürger können allenfalls an einzelnen Veranstaltungen teilnehmen, die vorrangig der Information dienen und nicht zur breiteren Interaktion einladen. Erste Ansätze für eine breitere Einbindung wurden allerdings mittlerweile begonnen. Im Rahmen des jährlich stattfindenden Forums organisierte die bayerische Präsidentschaft der Alpenraumstrategie einen Jugendkongress, der Vorschläge zur Stärkung der Jugendbeteiligung ausarbeitete. Die Aufnahme der Beiträge in die Umsetzung der Strategie bleibt allerdings offen. Daneben veranstaltete die Europäische Kommission einen Bürgerdialog, in dessen Rahmen Bürger Fragen zu europäischer Politik stellen konnten. Der partizipative Charakter des Bürgerdialogs war allerdings dadurch eingeschränkt, dass keine Entscheidungsträger der Alpenraumstrategie daran teilnahmen. Schließlich fand unter bayerischer Präsidentschaft erstmals ein Bürgerforum statt. Dabei handelt es sich um eine Ausstellung, in deren Rahmen sich die neun Aktionsgruppen präsentieren. Interessierte können sich an den Ständen über die Arbeit der Aktionsgruppen informieren, interaktive Anwendungen ausprobieren und mit den Aktionsgruppenleitern in Kontakt treten. Die Ausstellung bietet damit einen Ausgangspunkt für Gespräche und Interaktion abseits klassischer Frontalformate, ist allerdings nicht mit den Entscheidungsprozessen in der Alpenraumstrategie verbunden. Das Potential des Forums zur Demokratisierung hängt zudem von der Fähigkeit ab, einen breiten Kreis an Teilnehmern für die Interaktion zu gewinnen.

Ein großes Problem für Partizipation ist die Mobilisierung der Bevölkerung für alpenraumübergreifende Fragestellungen. Auch die Vertreter der

87 Christine Quittkat und Barbara Finke, »The EU Commission Consultation Regime«, in Beate Kohler-Koch, Dirk de Bièvre, und William Maloney (Hrsg.), Opening EU-Governance to Civil Society: Gains and Challenges (Universität Mannheim, Mannheimer Zentrum für Europäische Sozialforschung, 2008), 218. 
Regionen und Staaten sehen die Mobilisierung der Bürger als wichtige Herausforderung:

Die große Herausforderung wird sein, den kleinen Mann und die kleine Frau von der Straße in diesen Prozess einzubeziehen [...] Wirklich den Menschen das Gefühl zu vermitteln, dass diese EUSALP [European Union Strategy for the Alpine Region, EU-Alpenraumstrategie] etwas ist, das für sie passiert. Das wird die große Herausforderung werden, weil sonst die ganze EUSALP, ja, halt eine von tausenden und hunderten Organisationen ist, die es halt irgendwie gibt. ${ }^{88}$

Die Alpenraumstrategie ist bislang nicht in der breiteren Bevölkerung bekannt; damit sind auch Möglichkeiten, Bürger aktiv einzubeziehen, begrenzt. Grenzüberschreitende Kooperation schafft häufig keine Narrative und Aktivitäten, die Bürger begeistern und zur Teilnahme aufrufen, ${ }^{89}$ und knüpft weniger an die Lebenswelten der Bürger an. Politiken, die sich direkt und konkret auf ihre Interessen und Ziele auswirken, können allerdings eine Ausnahme bilden und zu punktuell starker Mobilisierung führen. ${ }^{90}$ Dabei spielt insbesondere die Kommunikation eine wichtige Rolle. Primäres Kommunikationsmittel der Alpenraumstrategie ist die Homepage, ${ }^{91}$ die allerdings wenige Informationen zur Verfügung stellt und diese nicht auf die Bevölkerung als Zielgruppe herunterbricht. Der geographisch große Alpenperimeter erschwert die Einbindung aller von einer Sachfrage betroffenen Beteiligten. In der Praxis müsste sich Partizipation daher auf geographische Teilbereiche oder Repräsentanten der Bevölkerung stützen. Eine weitere Schwierigkeit besteht in den unterschiedlichen Ressourcen und Fähigkeiten der Bürger, aktiv zu partizipieren. Wichtig sind hier Aktivitäten, die die Fähigkeiten der Bürger, sich selbst aktiv einzubringen, stärken..$^{92}$ Eine Möglichkeit, diese Fähigkeiten zu stärken, ist die Einbindung in öffentliche Debatten. Das Modell deliberativer Demokratie kann daher eine nützliche Ergänzung darstellen.

88 Interview mit einem Beamten einer österreichischen Region (Innsbruck, 27. Juli 2016).

89 Kramsch und Mamadouh, »Crossing Borders« (Fn. 7), 45.

90 Dahl, „Can International Institutions Be Democratic?«(Fn. 61), 30.

91 https:/www.alpine-region.eu/.

92 Sørensen und Torfing, »The Democratic Anchorage« (Fn. 35), 211. 


\subsection{Deliberative Demokratie}

Das Modell deliberativer Demokratie betont im Vergleich zur direkten und partizipativen Demokratie stärker die Dimension der Output-Legitimität. ${ }^{93}$ Ziel ist nicht die Beteiligung selbst, sondern die Schaffung effektiver und von allen mitgetragener Entscheidungen. Um diese Entscheidungen zu erreichen, sind Diskussionsprozesse notwendig, in denen sich alle betroffenen Akteure kommunikativ, rational und unter der Zuhilfenahme von argumentativen Begründungen austauschen. ${ }^{94}$ Am Ende dieses Diskussionsprozesses sollen sich alle Beteiligten gemeinsam auf ein Ergebnis einigen, das die vielfältigen Partikularinteressen in ein größeres Gemeinwohl einbettet. ${ }^{95}$

Die Input-Dimension deliberativer Demokratie betont, dass alle Betroffenen an Diskussionsprozessen teilnehmen sollen. Die Throughput-Dimension postuliert, dass alle Bürger gleiche Möglichkeiten haben sollen, Deliberation anzustoßen und ihre Meinungen einzubringen. Da Argumentation und nicht Machtausübung im Mittelpunkt steht, soll die Machtverteilung zwischen den Akteuren keine Rolle spielen. ${ }^{96}$ Auf der Output-Dimension dient der Prozess der Deliberation vor allem der Erarbeitung konsensfähiger Problemlösungen. Zugleich wird die Leistungsfähigkeit des politischen Systems erhöht. ${ }^{97}$ Die öffentliche, rationale, sachliche und konsensorientierte Artikulation, Erklärung und Rechtfertigung von Politik stärkt die Nachvollziehbarkeit von Ergebnissen, das Verantwortungsbewusstsein der Repräsentanten und damit Verantwortlichkeit und Kontrolle. ${ }^{98}$

Allerdings ist die idealtypische deliberative Situation, in der alle Teilnehmer auf gleicher Augenhöhe agieren, in der Realität aufgrund von unterschiedlichen Machtverhältnissen und Fähigkeiten schwer umsetzbar.

93 Blatter, »Demokratie und Legitimation« (Fn. 15), 277 f.

94 Vgl. Jürgen Habermas, Faktizität und Geltung. Beiträge zur Diskurstheorie des Rechts und des demokratischen Rechtsstaates (Suhrkamp, 1992); James Bohman und William Rehg (Hrsg.), Deliberative Democracy: Essays on Reason and Politics (MIT Press, 1997).

95 David Miller, "Deliberative Democracy and Social Choice«, (1992) 40 Political Studies 54.

96 Jack Knight und Jack Johnson, »What Sort of Equality Does Deliberative Democracy Require? «, in Bohman und Rehg (Hrsg.), Deliberative Democracy (Fn. 94).

97 Blatter, »Demokratie und Legitimation« (Fn. 15), $277 \mathrm{f}$.

98 Simone Chambers, »Deliberative Democratic Theory«, (2003) 18(6) Annual Review of Political Science, 308; Kratochwill und Fröhlich, »Legitimität und demokratische Potenziale« (Fn. 7), 71. 
Deliberation stellt gewisse Ansprüche an die Beiträge in deliberativen Prozessen; diese müssen rational, moderat und konsensfähig sein und das Gemeinwohl beleuchten. Beiträge, die nicht diesen Ansprüchen genügen, werden kaum berücksichtigt, da sie im Deliberationsprozess nicht artikulationsfähig sind. Bereits marginalisierte Gruppen können dadurch erneut ausgeschlossen werden. ${ }^{99}$ Daneben ist Partizipation von unterschiedlichen Fähigkeiten und Ressourcen der Betroffenen abhängig. ${ }^{100}$ Wie auch bei partizipativer und direkter Demokratie prägen zudem kommunikative Strategien und Medien Interessen und Diskussionen. ${ }^{101}$

Die Verbindung von grenzüberschreitender Kooperation und deliberativer Demokratie erscheint in der Literatur positiver als die Verbindung mit repräsentativen Modellen. ${ }^{102} \mathrm{Da}$ grenzüberschreitende Kooperation Arenen für verhandelte Entscheidungsprozesse schafft, die eine große Bandbreite an unterschiedlichen Akteuren und Perspektiven zusammenbringen, kann sie Deliberationsprozesse stimulieren. ${ }^{103}$ Die funktionale Definition von Beteiligung eröffnet Möglichkeiten, in transnationalen Kontexten, die sich durch Diversität, Interdependenz, Komplexität und die Unbeständigkeit von Problemstellungen auszeichnen, bessere Problemlösungen zu erzielen. ${ }^{104}$ Präferenzen der Bürger werden nicht nur aggregiert, sondern integriert, in einem Austausch kritisch überprüft und gegeneinander aufgewogen. ${ }^{105}$ Damit lässt sich deliberative Demokratie auch flexibler an sich verändernde Umstände und die Komplexität grenzüberschreitender Kooperation anpassen als repräsentative Modelle. ${ }^{106}$

Deliberation stößt zudem Lernprozesse an und beeinflusst die Präferenzen der beteiligten Akteure. Die gegenseitige Präsentation von Argumenten fördert den Willen und die Fähigkeit, ein Interesse am Interesse der anderen zu entwickeln. ${ }^{107}$ Solidarität, geteilte Werte und ein Sinn von

99 Lynn M. Sanders, »Against Deliberation«, (1997) 25 Political Theory 347.

100 Knight und Johnson, "What Sort of Equality« (Fn. 96).

101 Blatter, »Demokratie und Legitimation« (Fn. 15), 278.

102 Vgl. Kratochwill und Fröhlich, "Legitimität und demokratische Potenziale« (Fn. 7); Andreas Klinke, Demokratisches Regieren jenseits des Staates. Deliberative Politik im nordamerikanischen Große Seen-Regime (Barbara Budrich, 2006).

103 Eva Sørensen, „Governance and Democracy (Roskilde Universitet, 2010), 7 f.

104 Rainer Schmalz-Bruns, »Deliberativer Supranationalismus: Demokratisches Regieren jenseits des Nationalstaats«, (1999) 6 Zeitschrift für Internationale Beziehungen 185.

105 Bekkers und Edwards, »Legitimacy and Democracy«(Fn. 9), 70.

106 Sabel und Zeitlin, »Learning from Difference«; Hajer, »A Frame in the Fields«.

107 Miller, »Deliberative Democracy« (Fn. 95), 55. 
Gemeinschaft werden gefördert. ${ }^{108}$ Da es Bürgern häufig schwer fällt, die Präferenzen anderer, die weit entfernt leben oder einen völlig anderen Erfahrungshintergrund haben, zu verstehen, kann Deliberation als Brücke dienen. ${ }^{109}$ Kommunikation und Kollaboration können helfen, ein stärkeres Bewusstsein für Gemeinsamkeiten jenseits der eigenen nationalstaatlichen Sphäre zu schaffen und die Abgrenzung zwischen dem "Außen « und dem »Innen «, die konstitutiv für das Modell territorialer Repräsentation ist, aufzuweichen. ${ }^{110} \mathrm{Im}$ Gegensatz zum Modell territorialer Repräsentation muss damit auch keine gemeinsame Geschichte und Kultur konstruiert werden, um den Anspruch von Homogenität zu untermauern. Deliberative Demokratie stellt vielmehr die Integration von Differenz in den Vordergrund. ${ }^{111}$ Zugleich kann deliberative Demokratie das Modell territorialer Repräsentation ergänzen. Öffentliche Debatten erlauben es Bürgern, Wissen und Informationen über ein Thema zu erlangen und ihre Standpunkte im Dialog mit anderen Bürgern und Politikern zu überprüfen und kritisch zu bewerten. ${ }^{112}$

Die wichtigsten Foren für Deliberation in der Alpenraumstrategie sind die Entscheidungsgremien sowie die Aktionsgruppen, da hier die Entwicklung und Implementierung der Alpenraumstrategie diskutiert werden. Der Maßstab der Betroffenheit findet dahingehend Anwendung, dass der Alpenraum als funktionaler Bezugspunkt abgegrenzt wird. Damit nehmen potentiell nur jene Nationalstaaten und Regionen teil, die die Erfahrung des Lebens in Berggebieten teilen. Betroffene nichtstaatliche Akteure werden, wie bereits in den vorhergehenden Kapiteln ausgeführt wurde, allerdings kaum in Entscheidungen miteinbezogen. Zugleich umfasst die Alpenraumstrategie auch Vertreter von Regionen mit geringem Berganteil. Hinter der weiten territorialen Definition steht die Überlegung, die Wechselwirkungen zwischen dem Berggebiet und dem umliegenden Flachland sowie den großen Metropolen zu berücksichtigen. Der territoriale Perimeter wurde im Zuge politischer Verhandlungen abgegrenzt und hängt damit eng mit den Interessen der teilnehmenden Staaten und Regionen

108 Andreas Føllesdal, »Subsidiarity and Democratic Deliberation«, in Erik Oddvar Eriksen und John Erik Fossum (Hrsg.), Democracy in the European Union: Integration through Deliberation (Routledge, 2000), $90 \mathrm{f}$ and $105 \mathrm{f}$.

109 Dahl, »Can International Institutions Be Democratic?« (Fn. 61), 26.

110 Sørensen, »Governance Networks « (Fn. 42), $525 \mathrm{f}$.

111 Olivier Thomas Kramsch und Bohdana Dimitrovova, »T.H. Marshall at the Limit: Hiding out in Maas-Rhein Euregio«, (2008) 12 Space and Polity 31.

112 Sørensen, »Governance Networks « (Fn. 42), 511. 
zusammen. ${ }^{113}$ Deliberative Prozesse in der Alpenraumstrategie werden damit, so demokratisch sie auch sein mögen, durch die vorhergehenden Entscheidungen, die die Gemeinschaft definiert haben, mitbestimmt. ${ }^{114}$ In den neun Aktionsgruppen wiederum organisieren sich vor allem jene Verwaltungen, die sich von der jeweiligen Aktion betroffen fühlen. Allerdings ist die Teilnahme auch an Ressourcen und den politischen Willen, Personal bereitzustellen, gebunden. Ressourcenstarke Regionen nehmen an allen Aktionsgruppen teil, während kleinere Regionen ihre Teilnahme auf wenige Aktionsgruppen beschränken. Betroffene nichtstaatliche Akteure bleiben aus vielen Aktionsgruppen ausgeschlossen.

Die Entscheidungsgremien basieren auf dem Konsensprinzip; ${ }^{115}$ Entscheidungen sollen daher nur erfolgen, wenn alle Beteiligten sich hinter einer Einigung versammeln können. Damit ist ein wichtiges Prinzip deliberativer Demokratie angesprochen; Deliberation stützt sich nicht auf Mehrheitsentscheidungen, sondern auf das diskursive Finden gemeinsamer, für alle vorteilhafter Lösungen. Zugleich entsteht dabei Raum für Tauschgeschäfte, die der nationalen und regionalen Verteilungslogik folgen. Deliberation steht damit auch in einem Spannungsverhältnis mit dem klassischen Modell territorialer Repräsentation, da sich die teilnehmenden politischen Vertreter auch an den von ihnen vertretenen Wahlkreisen und nicht nur am gemeinsamen Interesse des Alpenraumes orientieren. In den Aktionsgruppen ist die Verteilungslogik schwächer ausgeprägt, da hier Vertreter der verschiedenen Verwaltungen Problemdefinitionen und mögliche Lösungen diskutieren. Durch die Abgrenzung zwischen mandatierten Entscheidungsträgern und nicht Entscheidungsberechtigten sind die Akteure allerdings direkter an ihre regionale und nationale Sphäre gebunden. Die Aktionsgruppen teilen die Deliberationssphäre zudem in sektorale Teilöffentlichkeiten, die sich etwa mit Verkehrs- oder Umweltthematiken beschäftigen, während Möglichkeiten zur Debatte über sektorale Grenzen hinweg noch wenig ausgeprägt sind. Hier gibt es allerdings mit Workshops, die von mehreren Aktionsgruppen gemeinsam gestaltet

113 Melanie Plangger, »Exploring the Role of Territorial Actors in Cross-Border Regions«, (2017) Territory, Politics, Governance 1, $12 \mathrm{ff}$.

114 Frederick G. Whelan, »Prologue: Democratic Theory and the Boundary Problem«, (1983) 25 Liberal Democracy 13.

115 Strategie der Europäischen Union für den Alpenraum, »Rules of Procedure for the Executive Board of the EU strategy for the Alpine Region « (Fn. 56); Strategie der Europäischen Union für den Alpenraum, »Rules of Procedure for the Action Groups of the EU strategy for the Alpine Region« (Fn. 56). 
werden, sowie gemeinsamen Projekten erste Ansatzpunkte für eine Überwindung der sektoralen Grenzen.

Ein weiteres Forum für Deliberation sind öffentliche, problemspezifische Workshops und Treffen, an denen potentiell jeder teilnehmen kann. Diese Workshops finden sowohl im Rahmen des jährlichen Forums der Alpenraumstrategie als auch in den einzelnen Aktionsgruppen statt. Die meisten Workshops folgen allerdings nicht deliberativen Maßstäben, sondern beinhalten vor allem Vorträge und Fragerunden, die wenig Raum für Argumentation und Verständigung bieten. Zudem ist die Teilnahme durch den geringen Informationsstand über die Strategie und die großen geographischen Distanzen limitiert. Eigene Bürgerdialoge und Bürgerforen im Rahmen des jährlich stattfindenden Forums sollen Deliberation befördern und für einen breiteren Personenkreis öffnen. Der Bürgerdialog wird von der Kommission organisiert, ist allerdings nur wenig an die Alpenraumstrategie gebunden, da Entscheidungsträger der Strategie nicht teilnehmen. Positiv anzumerken ist allerdings, dass die anwesenden Bürger selbst die Agenda bestimmen können, da sie im Vorhinein Themen und Fragen für die Debatte aufwerfen können. Das Bürgerforum stellt eher eine Möglichkeit zur ersten Information und Kontaktaufnahme dar, kann aber als Ausgangspunkt für den kommunikativen Austausch mit den Aktionsgruppen dienen.

Die makroregionale Alpenraumstrategie stellt das Modell deliberativer Demokratie vor besondere Herausforderungen. Voraussetzung für deliberative Demokratie im grenzüberschreitenden Kontext ist, dass transnationale Arenen für Deliberation geschaffen werden, die eine Partizipation am Willensbildungs- und Entscheidungsprozess ermöglichen. Dafür sind auch medial vermittelte transnationale Diskurse wichtig. Deliberation muss über etablierte institutionelle Strukturen hinweg schneiden und darf nicht in nationale oder regionale Sphären getrennt sein. In der Praxis erschweren allerdings auf den Nationalstaat ausgerichtete Medien und Kommunikationskanäle, Kulturen und Identitäten sowie Infrastrukturen und Rechtssysteme grenzüberschreitende Deliberation. ${ }^{116}$ Das territoriale Perimeter der Alpenraumstrategie umfasst sieben Staaten, 48 Regionen und rund 80 Millionen Menschen. ${ }^{117}$ Damit ist er für effektive Delibera-

116 Hans Knippenberg, »The Maas-Rhine Euroregion: A Laboratory for European Integration?«, (2004) 9 Geopolitics 608; Eeva-Kaisa Prokkola, »Cross-Border Regionalization and the Finnish-Swedish Border«, (2007) 36 Nordia Geographical Publications 15.

117 Europäische Kommission, »Mitteilung der Kommission zu einer Strategie der Europäischen Union für den Alpenraum«, 2. 
tionsprozesse, die alle Betroffenen einer bestimmten Fragestellung einbezieht, schlicht zu groß. Der Alpenraum ist zudem nach nationalstaatlichen und regionalen Trennlinien organisiert und in verschiedene Medienöffentlichkeiten geteilt. Daneben gibt es im Alpenraum vier große und mehrere kleinere Sprachgruppen.

Der Alpenraum dient damit kaum als Bezugspunkt für grenzüberschreitende Debatten, und die Bevölkerung kann, getrennt durch verschiedene Barrieren, Gemeinsamkeiten, geteilte Probleme und mögliche gemeinsame Lösungswege weniger durch Lernprozesse und Erlebnisse unmittelbar erfahren. Auch auf der Ebene der öffentlichen Verwaltungen fehlen oft Informationen über die Hintergründe in anderen Staaten und Regionen. Damit bleibt auch der Präferenzbildungsprozess auf die nationalstaatliche Sphäre beschränkt; grenzüberschreitende Interaktionsprozesse, die Lernen und Verständigung auslösen, können kaum entstehen. Auch ein Mangel an Transparenz und Öffentlichkeit schränkt deliberative Prozesse ein. ${ }^{118}$ Schließlich stellen auch Sprachbarrieren Deliberation vor praktische Schwierigkeiten. In der Alpenraumstrategie ist Englisch Arbeitssprache. Teilnehmer, die Schwierigkeiten haben, sich in der Fremdsprache Englisch auszudrücken, bleiben daher von Deliberationsprozessen ausgeschlossen.

Deliberative Prozesse müssten sich daher auf die Mikroebene und den Grenzraum zwischen zwei oder drei Alpenstaaten beschränken. In der Folge könnten diese Mikro-Prozesse in einen größeren, alpenraumübergreifenden Rahmen eingebettet werden. Um eine kognitive Basis zu schaffen, könnten Möglichkeiten für verschiedene Personengruppen geschaffen werden, Probleme, Potentiale und Herausforderungen der anderen Regionen und Staaten selbst zu erleben. Austauschprogramme und Exkursionen sind hier ein denkbarer Ansatz. Dabei müssen auch sprachgruppenübergreifende Möglichkeiten zur Artikulation geschaffen werden. Eine besondere Möglichkeit bieten dabei neue Medien, die virtuelle Interaktions- und Deliberationsräume schaffen. ${ }^{119}$ Allerdings setzt die Nutzung virtueller Medien voraus, dass die Entscheidungsträger den Alpenraum als virtuellen Deliberationsraum medial vermitteln und öffnen. Ein wichtiges Kommunikationsmittel sind dabei Internetseiten, die eine grenzüberschreitende Region symbolisch repräsentieren. ${ }^{120}$ Als virtueller Interaktionsraum bietet

118 Føllesdal, »Subsidiarity and Democratic Deliberation«(Fn. 108), 93.

119 Kramsch und Mamadouh, »Crossing Borders« (Fn. 7), 44.

120 Ebd., 45 f; Enza Lissandrello, »Cross-Border Region Espace Mont-Blanc: >A Territorial Not-Yet «? «, in Olivier Kramsch und Barbara Hooper (Hrsg.), Cross-Border Governance in the European Union (Routledge, 2004). 
sich zudem die Wissensplattform der Alpenraumstrategie an, die online Beiträge aller Interessierten sammeln und Dialogmöglichkeiten eröffnen kann.

Da die Möglichkeiten für deliberative Prozesse auf der makroregionalen Ebene bislang begrenzt und, aufgrund der geographischen Größe des Gebietes, auch schwierig umsetzbar sind, konzentriert sich Deliberation im Moment insbesondere auf die regionale und nationale Ebene. Nationale Koordinationsplattformen, die, wie etwa in Frankreich und Österreich, auch zivilgesellschaftliche Akteure einbeziehen, sowie Dialogveranstaltungen bieten dabei erste Ansatzpunkte für die Entstehung deliberativer Teilöffentlichkeiten, die alpenraumübergreifende Fragestellungen diskutieren. Eine Einbettung dieser Teilöffentlichkeiten in einen größeren geographischen Kontext wäre allerdings notwendig, um die deliberative Qualität der Alpenraumstrategie zu verbessern. Zudem erfordert effektive Deliberation ein Abkommen von klassischen Frontalvorträgen und die Einbindung in interaktive Formate, in denen jeder Themen auf die Agenda setzen und sich argumentativ einbringen kann.

Die Beteiligung breiter Bevölkerungsschichten, wie sie in direkten, partizipativen und deliberativen Demokratiemodellen vorgesehen ist, ist in grenzüberschreitender Kooperation noch sehr wenig vorhanden. Dies korrespondiert mit einer geringen Verankerung grenzüberschreitender Kooperation in den Erfahrungswelten der Bürger. ${ }^{121}$ Die praktischen Schwierigkeiten, vor denen das klassische Modell territorialer Repräsentation im grenzüberschreitenden Kontext steht, lassen eine Ergänzung mit Elementen anderer Demokratiemodelle allerdings sinnvoll erscheinen. Das folgende Kapitel stellt dazu abschließende Überlegungen an.

\section{Schlussfolgerungen}

Ziel des vorliegenden Beitrages war es, die demokratische Legitimität von grenzüberschreitender Kooperation im Kontext verschiedener Vor-

121 Jouni Häkli, »Governing the Mountains: Cross-Border Regionalization in Catalonia«, in Kramsch und Hooper (Hrsg.), Cross-Border Governance (Fn. 120), 56 ff; Anke Strüver, »Everyone Creates One's Own Bordersı: The Dutch-German Borderland as Representation", (2004) 9 Geopolitics 627; Anke Strüver, Stories of the >Boring Border: The Dutch-German Borderscape in People's Minds (LIT-Verlag, 2005); Juan-Manuel Trillo-Santamaría, »Cross-Border Regions: The Gap Between the Elite's Projects and People's Awareness. Reflections from the Galicia-North Portugal Euroregion«, (2014) 29 Journal of Borderlands Studies 257. 
stellungen von Demokratie zu untersuchen. Als empirisches Fallbeispiel diente dabei die makroregionale Strategie für den Alpenraum, an der Politiker und Beamte aus 48 Regionen und sieben Staaten teilnehmen. Da diese potentiell weitreichende Auswirkungen auf die Stellung der politischen Akteure, EU-, nationale und regionale Politiken sowie die Allokation von öffentlichen Geldern hat, steht auch die Alpenraumstrategie vor der Herausforderung, demokratische Legitimität für grenzüberschreitendes Handeln zu schaffen.

Verschiedene Modelle von Demokratie haben dabei sehr unterschiedliche Vorstellungen, was demokratische Legitimität im grenzüberschreitenden Kontext beinhalten muss. Demokratiemodelle dienen immer nur der Annäherung an ein normatives Ideal, können allerdings aufzeigen, wo etablierte Formen der Legitimität vor besonders großen Hindernissen stehen. Das weitverbreitetste Modell zur Bewertung demokratischer Legitimität ist das Modell territorialer Repräsentation, das Legitimität an die Vertretung territorialer Wahlkreise bindet. Allerdings stößt dieses Modell im grenzüberschreitenden Kontext an Grenzen. Die Bindung der Entscheidungsträger an die eigenen nationalstaatlichen oder regionalen Wahlkreise unterminiert Repräsentation, schwächt die grenzüberschreitende Region als Ganzes und schafft Verteilungskonflikte. Die Schaffung eines neuen grenzüberschreitenden Wahlkreises wiederum hätte Konflikte mit bestehenden Wahlkreisen zur Folge. Das Modell der funktionalen Repräsentation, das Legitimität über den freien Wettbewerb von Interessengruppen herstellt, ist dadurch limitiert, dass Interessengruppen über verschiedene Ressourcen und einen unterschiedlichen Organisationsgrad verfügen. In der Praxis fungieren öffentliche Akteure häufig als Gatekeeper, die Interessengruppen allerdings kaum nach objektiven Kriterien auswählen. Die intern häufig nicht demokratischen Strukturen von Interessengruppen und fehlende Mechanismen zur Integration von Interessen unterminieren zudem die Verantwortlichkeit.

Auch Demokratiemodelle, die Teilhabe stärker in den Vordergrund stellen, stehen vor vielen Schwierigkeiten im grenzüberschreitenden Kontext. Direkte Demokratie bei grenzüberschreitender Kooperation könnte vor allem mit der Einführung grenzüberschreitender Abstimmungen zu Sachfragen umgesetzt werden, wird allerdings durch die gesetzliche Bindung von Abstimmungen an den Nationalstaat oder die administrative Region limitiert. Auch der Mangel an transnationaler Öffentlichkeit schränkt direkte Demokratie ein. Partizipative Ansätze scheitern häufig an der mangelnden Fähigkeit zur Mobilisierung breiter Bevölkerungsschichten und dem Fehlen von konkreten Partizipationsangeboten. Deliberative Ansätze könnten viele Mängel anderer Demokratiemodelle ausgleichen. Sie zielen auf die 
Schaffung einer gemeinsamen Verständigungsbasis ab, stellen argumentative Diskussion in den Vordergrund und umgehen damit die exklusive nationalstaatliche Logik. Zugleich ist grenzüberschreitende Deliberation in der Praxis schwer umsetzbar. Sie setzt grenzüberschreitende Debatten, die Abwesenheit von asymmetrischen Machtverhältnissen und den effektiven Einbezug aller Beteiligten in Willensbildungs- und Entscheidungsprozesse voraus. Das Beispiel der Alpenraumstrategie zeigt, dass diese Voraussetzungen in der Praxis kaum gegeben sind.

Grenzüberschreitende Kooperation steht vor der Herausforderung, die Bevölkerung mitzunehmen und stärker einzubinden. Ohne eine stärkere Verankerung in der Bevölkerung bleibt Kooperation auf die Zusammenarbeit öffentlicher Institutionen beschränkt, während sie nicht in das Bewusstsein und die Identitäten der Bürger vordringt. Grenzüberschreitende Kooperation muss daher Mechanismen schaffen, um Beiträge der Bevölkerung in Entscheidungen und Handlungen einzuspeisen, ihre Berücksichtigung im Entscheidungsprozess zu gewährleisten und zu ermöglichen, dass Entscheidungsträger verantwortlich gehalten werden. Dabei lässt sich der nationalstaatliche Maßstab von Demokratie nicht einfach auf die grenzüberschreitende Sphäre übertragen. Vielmehr scheint die Entwicklung neuer Formen der Legitimität, die sich partizipativer oder deliberativer Elemente bedienen, im grenzüberschreitenden Kontext notwendig. Entscheidungsträger sollten Kooperation daher in Zukunft als Ausgangspunkt nehmen, um verschiedene Formate auszuprobieren und Prozesse für unterschiedliche Personengruppen zu öffnen. Besonderer Wert sollte dabei auf Kommunikation, die Entwicklung grenzüberschreitender Narrative, die Festlegung transparenter Prozeduren und Kriterien zur Auswahl der Agenda und der Teilnehmer sowie die Schaffung transnationaler öffentlicher Arenen gelegt werden. Grenzüberschreitende Kooperation kann damit nicht nur zu einem Feld für neue Problemlösungen, sondern auch zum Experimentierfeld für neue Formen demokratischer Legitimität werden. 
